\title{
Thermal network model and experimental validation for a motorized spindle including thermal- mechanical coupling effect
}

Changjiang Zhou

Hunan University

Zefeng Qu

Hunan University

Bo Hu ( $\sim$ hubo4956@csust.edu.cn )

Changsha University of Science and Technology https://orcid.org/0000-0002-2513-4342

Shengbo Li

Xiamen University of Technology

\section{Research Article}

Keywords: Motorized spindle, Thermal network model, Thermal-mechanical coupling, Temperature field

Posted Date: February 23rd, 2021

DOl: https://doi.org/10.21203/rs.3.rs-159145/v1

License: (a) (i) This work is licensed under a Creative Commons Attribution 4.0 International License.

Read Full License 


\title{
Thermal network model and experimental validation for a motorized
}

\section{spindle including thermal-mechanical coupling effect}

\author{
Changjiang Zhou ${ }^{1}$, Zefeng $\mathrm{Qu}^{1}$, Bo $\mathrm{Hu}^{1,2^{*}}$, Shengbo $\mathrm{Li}^{3}$ \\ 1. State Key Laboratory of Advanced Design and Manufacturing for Vehicle Body, Hunan University, Changsha 410082, P. R. China \\ 2. Hunan Provincial Key Laboratory of Intelligent Manufacturing Technology for High-performance Mechanical Equipment, \\ Changsha University of Science and Technology, Changsha 410114, P. R. China \\ 3. College of Mechanical and Vehicle Engineering, Xiamen University of Technology, Xiamen 361024, P. R. China
}

\begin{abstract}
Thermal deformation caused by temperature rise have an influence on the dynamic performance of a motorized spindle. In turn, the change in the dynamic performance will affect the temperature rise and thermal deformation of the system. However, the latter was rarely focused on in the previous literature. Therefore, a thermal network model of motorized spindle is enhanced by considering the thermal-mechanical coupling effect. Then, an iterative method is presented to solve the coupled equations, and a temperature test rig of the motorized spindle is set up to verify the proposed model. The relative error between the predicted and experimental results at two test points decreases by $9.56 \%$ and $3.44 \%$ after considering the thermal-mechanical coupling effect. The comparison with the experimental results shows that the proposed model with thermal-mechanical coupling effect can obtain a more accurate temperature field than the previous model.
\end{abstract}

Key words: Motorized spindle; Thermal network model; Thermal-mechanical coupling; Temperature field

\section{Introduction}

Motorized spindle is a core functional component of computer numerical control (CNC). High transmission accuracy is implemented by the spindle system because the spindle is driven by the motor directly. However, the thermal deformation of the spindle system, caused by uneven temperature rise, reduces machining precision and reliability of a motorized spindle [1]. Temperature affects not only the execution accuracy of the mechanical system [2], but also the machining accuracy of the machine tool [3]. To compensate for the error induced by the thermal deformation, an accurate temperature field prediction model of the motorized spindle is essentially studied.

The temperature rise of the spindle system usually focuses on thermal characteristics. To date, considerable efforts have been dedicated on the thermal characteristics of the spindle system. As the bases of calculation for the temperature in the spindle system, a thermal-dynamic model of bearings was established by Palmgren [4] and the frictional heat of bearings was calculated by Jones [5]. Thereafter, the thermal characteristics of the spindle system have attracted increasing attention. The temperature in the spindle system was determined through the finite difference model (FDM) by Harris [6], but the thermal deformation of the spindle system was not included in their model. Later, a thermal characteristic model with thermal deformation was developed [7]. The finite element method (FEM) was also used to predict the temperature field of the spindle system [8-10].

Recently, motorized spindles as a core part of $\mathrm{CNC}$ have been concerned. A finite difference model with heat transfer mechanism of motorized spindle was proposed by Bossmanns [11], and the temperature at several locations matched the measured values. Chen et al. [12] developed a thermal error model of motorized spindle and found that spindle temperature has the characteristics of time-varying, nonlinearity, and strong dependence on rotation speed. Affected by these factors, the thermal characteristics of motorized spindles are considerably complicated. Afterward, an integrated thermomechanical coupled dynamic model of motorized spindle was developed. Li et al. [13,14] predicted the temperature of the spindle and bearings at different preloads, moments and speeds. The analysis results showed that the predicted temperature matched the measured one, except for the temperature of the shell near the motor. Chen [15] analyzed the effects of speed, bearing configuration, and coolant flow on the temperature through a temperature rise experiment. The result indicated that reasonable bearing configuration, low-viscosity lubricating oil, and high cooling water flow are important for the thermal performance of the motorized spindle. FEM was 
gradually adopted and developed in temperature prediction of spindle systems. Lee et al. [16] analyzed the temperature and thermal deformation of the spindle system, and the results showed that the convection has to be considered. Zivkovic et al.[17] investigated the interaction between temperature and bearing stiffness. The results showed that the bearing stiffness of spindle system is enhanced with an increase in rotation speeds. Combined the FEM and the conjugate gradient method, the time-varying heat sources in the high speed spindle are successfully estimated by Than et al. [18]. Their results show that this method can be used for reference in thermal compensation design. To decrease the thermal error caused by temperature rise, Yang et al. [19] developed the FEM model by using design of experiment. Liu et al. [20] established a thermal-structure model by considering bearing thermal preload. Zhang et al. [21] proposed a thermal deformation prediction model for the 100 MD60Y4 motorized spindle by optimizing the heat transfer coefficient. Yan et al. [22] investigated the variation rules of the spindle temperature field, and found that the variation process can be divided into the non-uniform and uniform variation stages. Their findings can provide a new perspective for temperature variable selection and coefficients adjustment. In addition, more factors were taken into account in the predicted model of temperature filed, such as cooling and lubrication [23,20], thermal contact resistance (TCR) [24-27,20], heat transfer coefficient [28], dynamic effect [29,30], etc.

In addition to the complicated FDM and FEM, a thermal network model based on thermal resistance has recently been applied to predict the temperature field of the spindle system. Liu et al. [31] developed a thermal resistance network model of spindle-bearing-pedestal for motorized spindle and analyzed the effects of thermal resistance on the steady temperature field. Nevertheless, the importance of time-varying thermal parameters for motorized spindle analysis was underrated in the model. A thermal deformation coupling model in thermal network for the spindle-bearing system was studied by Yan et al. [32]. In this model, other time-varying parameters, such as thermal deformation and lubrication viscosity, were considered, and the temperature of bearing outer ring was approximate to the measured result at mid and high speed. Considering the effect of forced vibration on bearing heat generation, the bearing heat generation model was improved by Zheng et al. [33]. The bearing temperature rise can be better forecasted by employing this model. Furthermore, the influence of cooling system on the temperature variation of motorized system has captured the attention of researchers. Liu et al. [34] developed an improved thermal resistance network model by considering the temperature variation of cooling system and analyzed the effects of coolant flow rate and speed on the temperature variation of the spindle system. The results indicated that the coolant flow rate has a considerable effect on the temperature of bearings at high rotation speeds.

The abovementioned works have enriched the research on the thermal dynamic characteristics of motorized spindles and are of great significance to the improvement of their thermal dynamic performance. In fact, the temperature and dynamic performance of a motorized spindle are coupled and inseparable and include two points: (1) the thermal deformation caused by temperature rise will affect the dynamic performance of the motorized spindle; (2) the change in the dynamic performance will in turn affect the temperature rise and thermal deformation of the system. However, the mentioned works have mainly focused on content (1), whereas content (2) has rarely been covered. Therefore, an enhanced thermal network model for a motorized spindle is proposed in this work by considering the thermal-mechanical coupling effect. A new method for solving bearing heat is given, in which the effect of temperature rise is considered. A temperature experiment is performed to verify the proposed model.

The rest of this paper is organized as follows. In Section 2, the thermal network model for motorized spindle is established, and a new method for solving bearing heat is provided. In Section 3, the computation flowchart is given to acquire the steady temperature field of the motorized spindle, and a comparison analysis with the experimental results is performed. Thermal displacement, contact angle, contact load, friction heat, and temperature for bearings are investigated in Section 4 to interpret the change law of temperature with speeds. Lastly, the conclusions are indicated in Section 5.

\section{Model}

\subsection{Geometric model}

The three-dimensional geometry model of a motorized spindle is shown in Fig. 1. Bolts, bolt holes, cooling 
pipes, and lubrication pipes are omitted, and some small chamfers and rounded corners are simplified. The rotor is connected with the rotating shaft by pressure matching, and the helical cooling jacket is fitted on the stator surface. Angular contact ball bearings are utilized to support the rotating shaft. Herein, three B7018C bearings are installed at the front of the shaft, and one B7011C bearing is installed at the rear of the shaft, as shown in Fig. 1. The heat generated by the stator is carried away by the coolant in the helical cooling jacket, and part of the friction heat in the bearing raceway is taken away by grease.

The motorized spindle is axisymmetric; hence, its thermal characteristics can be analyzed by establishing a two-dimensional heat transfer structure. In Fig. 2, the red lines with arrows represent heat transfer paths, and the heat diffuses outward to the environment in the arrow direction. Points $A$ and $B$ represent test points 1 and 2 near the outer ring of bearing presented in Section 3.2, respectively.

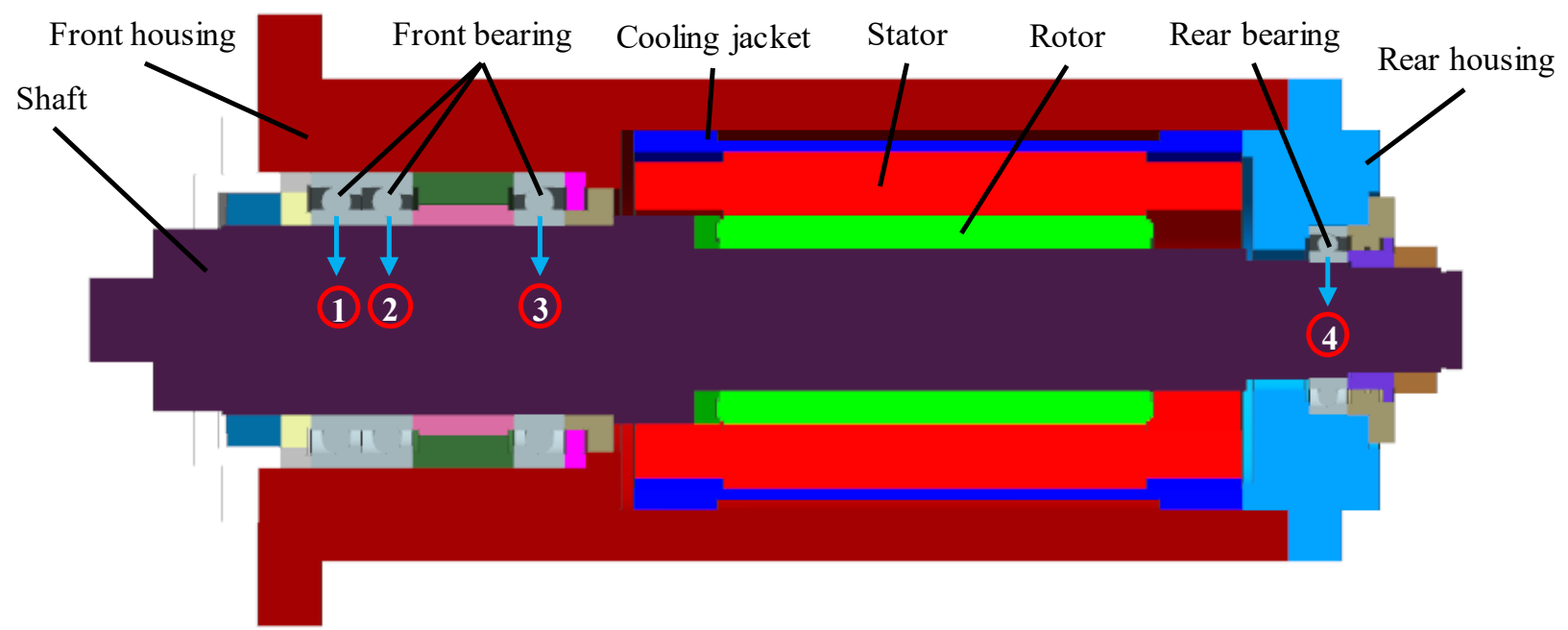

Fig. 1 Geometric model of motorized spindle

The thermal network model based on thermal resistance is established through the following steps: (1) In accordance with the number of balls in bearing, the motorized spindle is equally divided along the circumference; (2) In accordance with the heat transfer mechanism, the thermal network of the motorized spindle is established; (3) The system thermal equilibrium equations are derived using Kirchhoff's current law (KCL); (4) The steady temperature field of the motorized spindle is obtained by coupling the equations of temperature field and bearing heat.

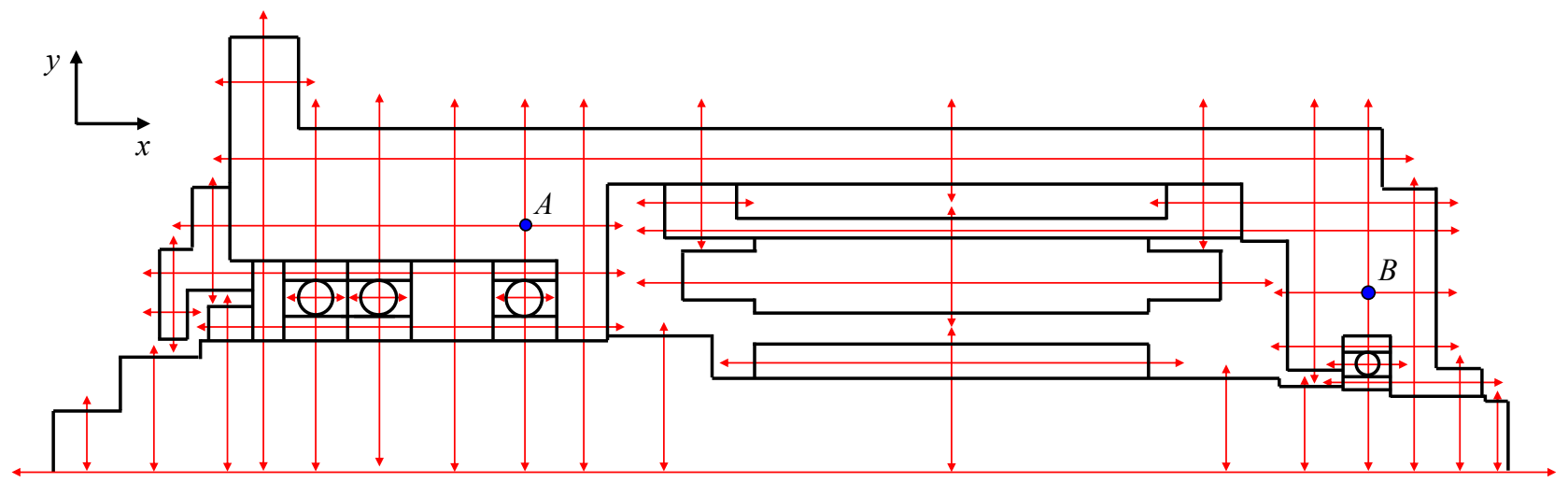

Fig. 2 Heat transfer path of motorized spindle

\subsection{Thermal network model}

In accordance with the heat transfer mechanism of motorized spindle, the global (Fig. 3) and local (Fig. 4) thermal network models are established based on thermal resistance. In these models, the thermal resistance of the motorized spindle mainly includes the thermal convection resistance of the solid and environmental fluid, the thermal conduction resistance inside the solid, and the TCR among contact surfaces. 


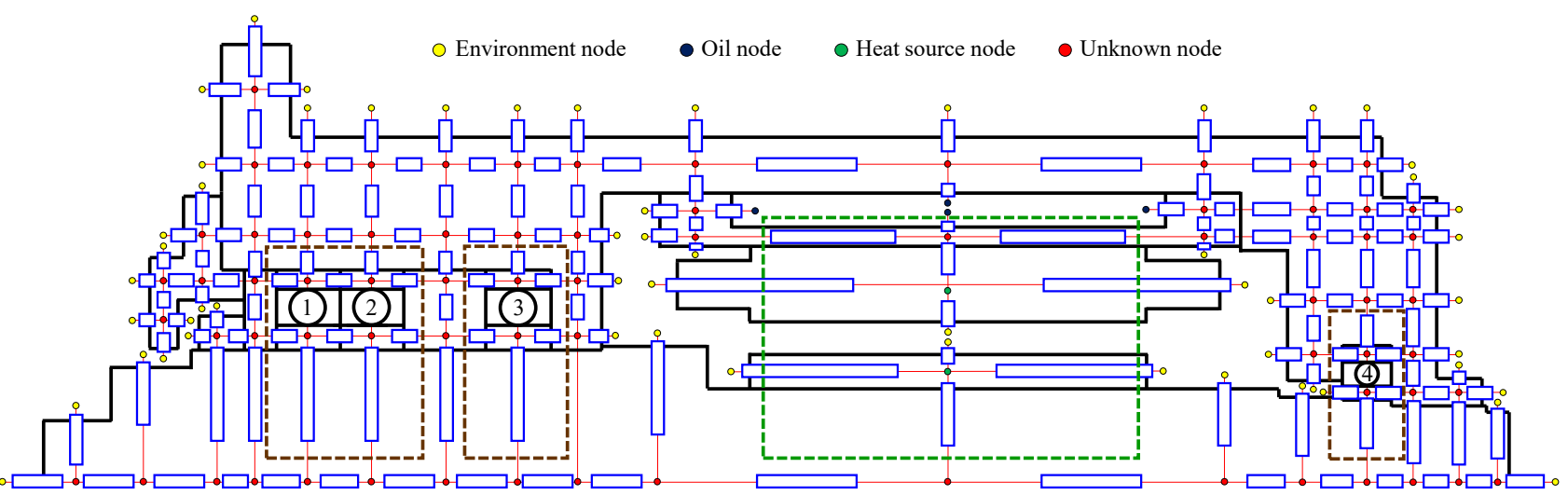

Fig. 3 Global thermal network model of motorized spindle

As shown in Fig. 4 a, the thermal resistance $R_{b}$ of ball and $R_{L i}$ or $R_{L o}$ of grease are connected in parallel for grease ball bearing. $H_{i}$ and $H_{o}$ respectively represent the frictional heat generated by the inner raceway and outer raceway of the bearing, and the heat is transferred outward through the thermal resistance. $R_{i s}$ and $R_{o h}$ denote the TCR between the bearing inner ring and the spindle and between the bearing outer ring and the bearing housing, respectively. In Fig. $4 \mathrm{~b}, R_{r s}$ and $R_{s c}$ respectively represent the TCR between the rotor and spindle and between the stator and cooling pipe. The heat $H_{s}$ of the stator can be greatly removed using the oil in the cooling pipe and the turbulent gas in the air gap of the motor, and the heat $H_{r}$ of the rotor is mainly taken away by the rotor rotation effect.

(a)

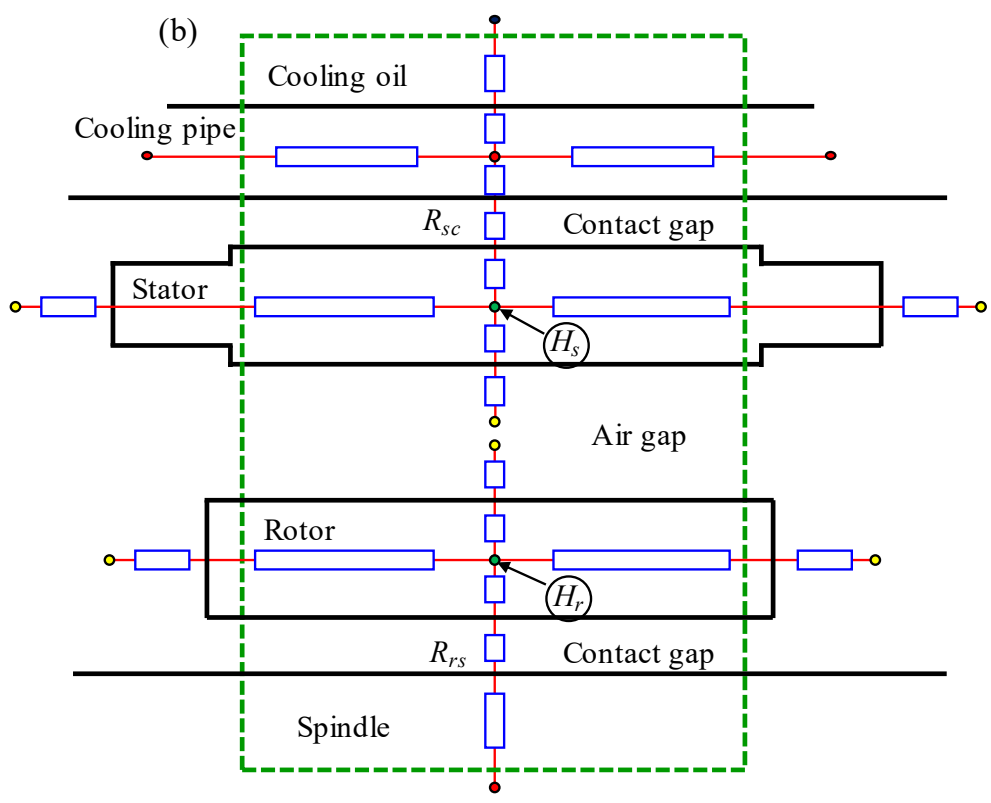

Fig. 4 Local thermal network model for (a) bearings and (b) motor
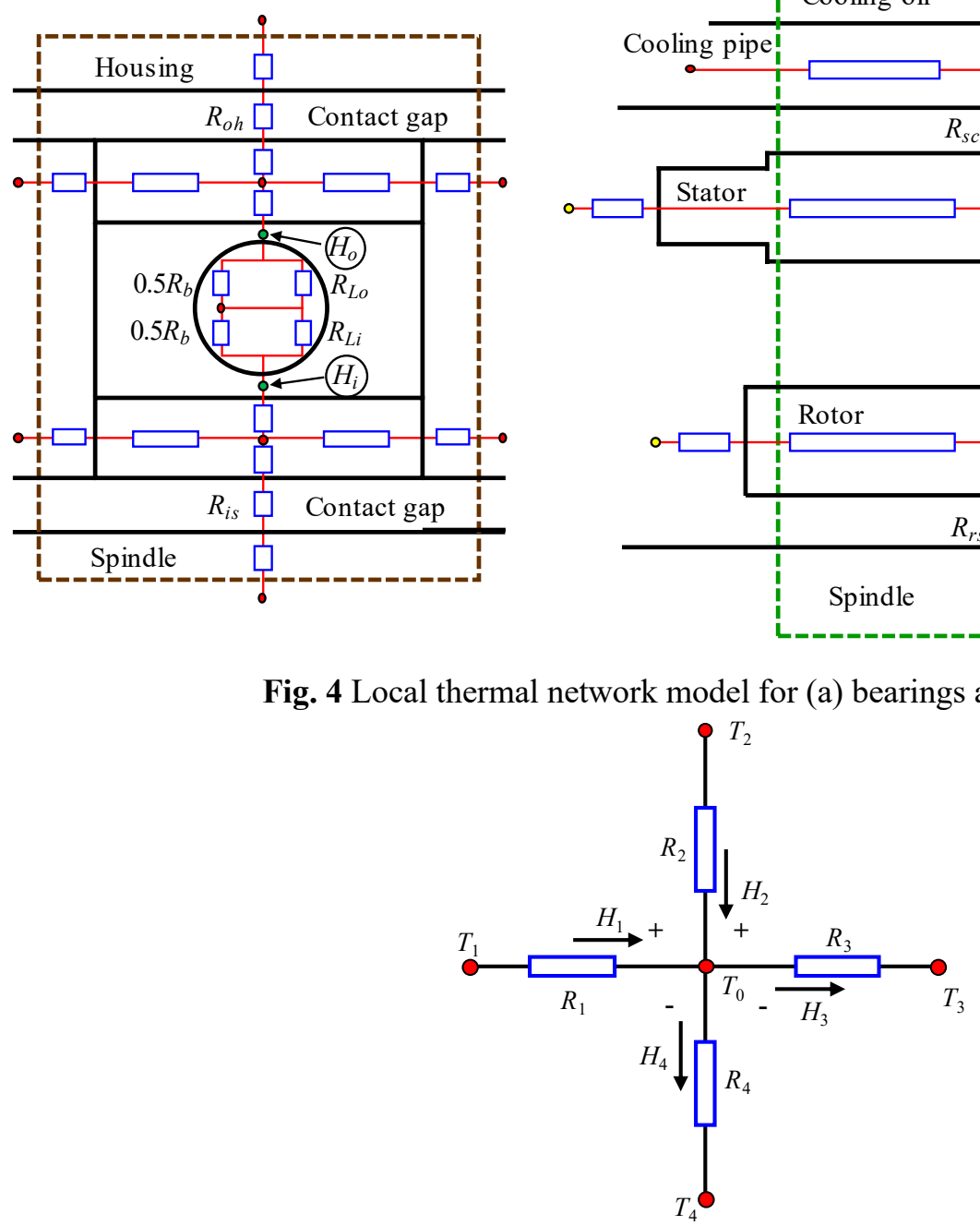

Fig. 5 Heat transfer diagram 
As shown in Fig. 5, KCL with thermal resistance can be applied to solve the thermal network model of motorized spindle. On the basis of KCL, the heat flow into a node is positive; otherwise, it is negative. The algebraic sum of all the heat flow involved in the node is equal to zero, and it can be expressed as

$$
\sum H=0,
$$

$N$ temperature nodes are assumed to exist in the system, and each node is connected with $m$ heat transfer routes. The thermal equilibrium equation of the nodes is written as

$$
H_{i}+\sum_{j=1}^{m_{i}} \frac{T_{j}-T_{i}}{R_{i j}}=0,
$$

where $i, j=1,2, \ldots, N ; T_{i}$ and $T_{j}$ denote the temperatures of nodes $i$ and $j$, respectively; $R_{i j}$ represents the thermal resistance between nodes $i$ and $j$; and $H_{i}$ describes the thermal flow generated by node $i$, which is regarded as the inflow thermal flow.

The thermal equilibrium equations of all nodes can be expressed in the following matrix form

$$
[\mathbf{H}]+[\mathbf{R}]^{-1}[\mathbf{T}]=\mathbf{0},
$$

where $[\mathbf{H}]$ denotes the heat flow matrix, which is determined using bearing friction and motor loss; $[\mathbf{R}]$ represents the thermal resistance matrix, and [T] describes the temperature distribution matrix.

The thermal network parameters include the heat flow matrix $[\mathbf{H}]$, thermal resistance matrix $[\mathbf{R}]$, and temperature distribution matrix $[\mathbf{T}]$. $[\mathbf{T}]$ can be determined using $[\mathbf{H}]$ and $[\mathbf{R}]$. $[\mathbf{H}]$ includes the heat generated by bearing and motor, and $[\mathbf{R}]$ includes the thermal conduction resistance, thermal convection resistance, and TCR. Under the influence of the cooling system, the effect of thermal radiation on the temperature field can be ignored for the motorized spindle.

In this work, the bearing housing, inner ring, outer ring, stator and rotor of the motor are simplified into a hollow cylinder, while the solid spindle is simplified into a cylinder. The thermal conduction resistance can be divided into axial and radial resistance. From the Fourier theorem, the axial thermal conduction resistance of the cylinder is given as

$$
R_{1}=\frac{L}{k S_{a}},
$$

where $L, k$, and $S_{a}$ denote the axial length, thermal conductivity and axial cross-sectional area of the cylinder, respectively.

The radial thermal conduction resistance of the hollow cylinder is expressed as

$$
R_{2}=\frac{\ln \left(d_{2} / d_{1}\right)}{2 \pi k L},
$$

where $d_{1}$ and $d_{2}$ are the inner and outer diameters of the cylinder, respectively.

The radial thermal conduction resistance of a solid cylinder can be written as

$$
R_{3}=\frac{1}{\pi k L} .
$$

The thermal conduction resistance of the ball is expressed as

$$
R_{4}=\frac{2}{\pi k_{b} D_{b}},
$$

where $k_{b}$ is the thermal conductivity of the ball.

The thermal conduction resistance of grease is described as

$$
R_{5} \approx \frac{D_{b}}{2 k_{L}\left(\pi d_{i} B_{i}-\frac{1}{4} \pi Z D_{b}^{2}\right)},
$$




$$
R_{6} \approx \frac{D_{b}}{2 k_{L}\left(\pi D_{o} B_{o}-\frac{1}{4} \pi Z D_{b}^{2}\right)},
$$

where $k_{L}$ is the thermal conductivity of grease; $d_{i}$ and $D_{o}$ denote the diameters of the bearing inner and outer raceways, respectively; and $B_{i}$ and $B_{o}$ represent the width of the bearing inner and outer rings.

According to Fourier theorem, the axial and radial thermal convection resistance for a cylinder is written as

$$
R_{7}=\frac{1}{h S}
$$

where $h$ denotes the convective heat transfer coefficient, and $S$ is the area of convective heat transfer between the cylinder and fluid.

The convective heat transfer coefficients on the surfaces of the motorized spindle can be expressed as

$$
h_{1}=\frac{N_{u} \lambda}{D_{h}},
$$

where $N_{u}$ and $\lambda$ denote the Nusselt number and fluid thermal conductivity, respectively; and $D_{h}$ is the geometric characteristic dimension of the heat exchange.

For highly turbulent flow, $N_{u}$ is described as

$$
N_{u}=0.0225 R_{e}^{0.8} P_{r}^{0.3},
$$

where $P_{r}$ is the Prandtl number; and $R_{e}$ denotes the Reynolds number, which can be written as

$$
R_{e}=\frac{u D_{h}}{\eta_{f}},
$$

where $u$ is the average velocity of fluid, and $\eta_{f}$ is the kinematic viscosity of fluid.

The forced convection heat transfer coefficient between the surface of parts and the fluid can be given as

$$
h_{2}=28\left(1+\sqrt{\frac{0.225 \omega_{s} d_{e}}{p}}\right),
$$

where $\omega_{s}$ denotes the rotating speed of spindle, $d_{e}$ is the equivalent diameter of the rotating surface, and $p$ refers to the pole pairs of motor.

During the operation of the motorized spindle, the composite heat transfer coefficient between the bearing housing and the environmental fluid is described as [35]

$$
h_{3}=9.7\left(\mathrm{~W} /\left(\mathrm{m} \cdot{ }^{\circ} \mathrm{C}\right)\right)
$$

The actual contact between the mating mechanical surface only occurs in the finite asperities on the contact surface. Hence, as the heat passes through the interfacial clearance, additional resistance is produced, which is defined as TCR. The TCR is written as

$$
R_{8}=\frac{1}{h_{c} A},
$$

where $h_{c}$ denotes the contact thermal conductivity of fluid in interfacial clearance, and $A$ is the nominal contact area between the mating mechanical surfaces.

In consideration of the thermal conduction effect of asperities and interfacial fluid medium, the contact thermal conductivity is expressed as [24]

$$
h_{c}=\frac{1}{L_{g}}\left[A^{*}\left(\frac{2 k_{1} k_{2}}{k_{1}+k_{2}}\right)+\left(1-A^{*}\right) k_{f}\right] \text {, }
$$

where $L_{g}$ is the thickness of the interfacial clearance; $k_{1}$ and $k_{2}$ denote the thermal conductivity of the mating components; $k_{f}$ represents the thermal conductivity of the interfacial clearance medium; $A^{*}$ is the dimensionless actual contact area [24], and $A^{*}=A_{c} / A, A_{c}$ denotes the actual contact area. 


\subsection{Computation method of Heat}

As an important part of the motorized spindle, the built-in permanent magnet synchronous motor has the characteristics of high efficiency, small volume, light weight, and low temperature rise. In accordance with classical core loss separation theory, the losses of the built-in permanent magnet synchronous motor mainly include the stator winding copper, iron, and rotor eddy current losses. The stator winding copper loss is determined using the resistance of the stator winding and the current in the winding, and it can be written as

$$
H_{C u}=m I_{s}^{2} R_{s} \text {, }
$$

where $H_{C u}, m, I_{s}$, and $R_{s}$ denote the copper loss, phase number, current, and resistance of stator winding, respectively.

From the classical model of core loss separation [36], the core loss caused by any magnetic density distribution includes hysteresis, eddy current, and additional losses, which can be expressed as

$$
H_{F e}=H_{h}+H_{e}+H_{e x c} \text {. }
$$

In Eq. (19), $P_{h}, H_{e}$, and $H_{e x c}$ can be described as

$$
\begin{gathered}
H_{h}=M_{s}\left(k_{h} f B_{m}^{\alpha}\right), \\
H_{e}=M_{s}\left(k_{e} f^{2} B_{m}^{2}\right), \\
H_{e x c}=M_{s}\left(k_{e x c} f^{1.5} B_{m}^{1.5}\right),
\end{gathered}
$$

where $H_{F e}$ denotes the core loss; $H_{h}, H_{e}$, and $H_{\text {exc }}$ represent the hysteresis, eddy current, and additional losses, respectively; $M_{s}$ and $f$ are the stator mass and working frequency of the motor, respectively; $k_{h}, k_{e}$, and $k_{\text {exc }}$ are the coefficients of hysteresis, eddy current, and additional losses, respectively; $B_{m}$ is the maximum magnetic flux density; and $\alpha$ is the dimensionless hysteresis loss parameter.

The rotor eddy current loss of the permanent magnet synchronous motor is expressed as

$$
H_{r}=\rho_{r} l \iint J^{2} d s,
$$

where $H_{r}$ denotes the eddy current loss of rotor; $\rho_{r}$ and $l$ are the resistivity of the rotor material and the axial length of the rotor, respectively; $J$ denotes the induced eddy current density; and $s$ represents the cross-sectional area of the rotor.

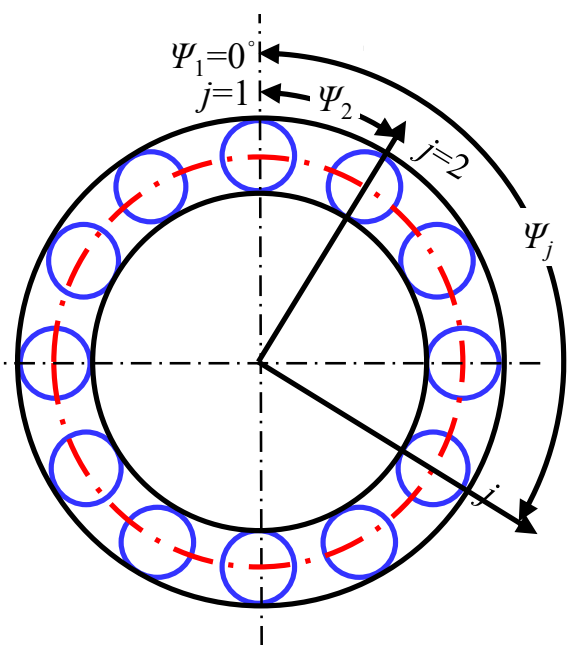

Fig. 6 Position angle of balls

As a crucial parameter in the thermal network model, the frictional heat generated by bearing should be considered and calculated accurately. The definition of position angle for bearing balls is shown in Fig. 6, and the position of ball, inner and outer rings before and after loading is illustrated in Fig. 7. Without loss of generality, the curvature center of the bearing outer raceway is assumed to be fixed in this work. Under the action of force $\mathbf{F}=\left[F_{x}\right.$, $\left.F_{y}, F_{z}, M_{x}, M_{y}\right]$, the relative displacement $\boldsymbol{\delta}\left(\boldsymbol{\delta}=\left[\delta_{x}, \delta_{y}, \delta_{z}, \theta_{x}, \theta_{y}\right]\right)$ in the bearing occurs. In consideration of frictional 
heat, the thermal displacement $\mathbf{u}\left(\mathbf{u}=\left[u_{a}, u_{r}\right]\right)$ in the bearing is generated. At high speed, the centrifugal displacement $u_{c}$ is produced by the bearing.

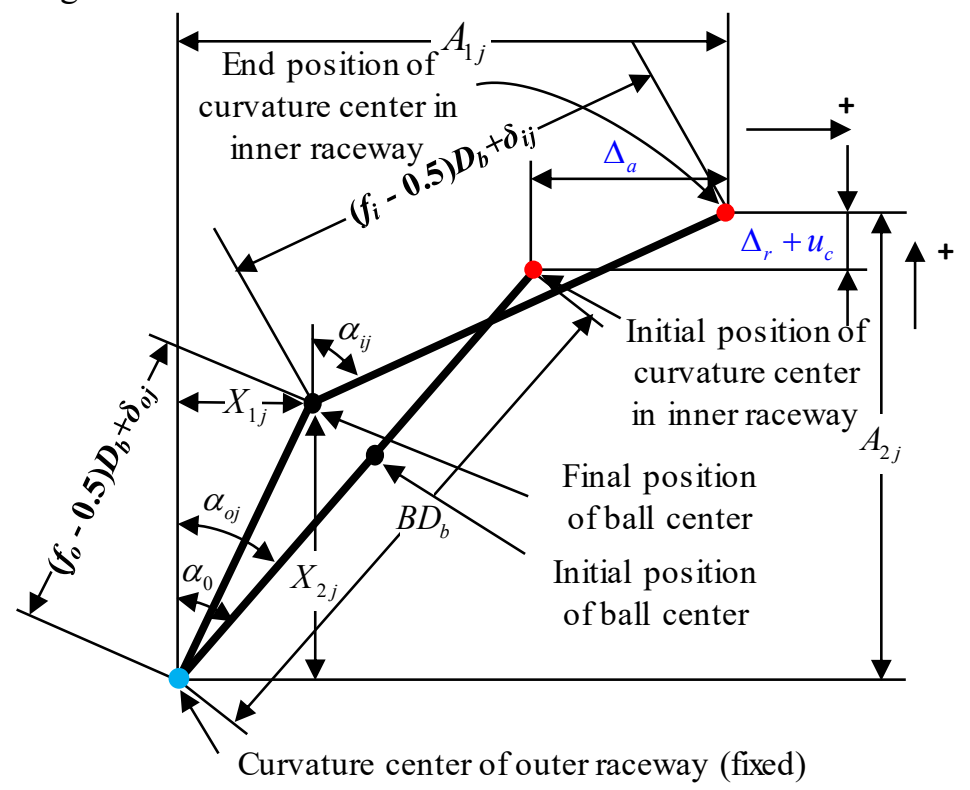

Fig. 7 Position of ball, inner and outer rings before and after loading

As shown in Fig. 7, at the position angle $\Psi_{j}$, the relative axial distance $A_{l j}$ and radial distance $A_{2 j}$ between the bearing inner and outer raceways can be expressed as follows:

$$
\begin{gathered}
A_{1 j}=B D_{b} \sin \alpha_{0}+\Delta_{a}, \\
A_{2 j}=B D_{b} \cos \alpha_{0}+\Delta_{r}+u_{c},
\end{gathered}
$$

where the parameter $B$ is written as $B=f_{i}+f_{o}-1, f_{i}$ and $f_{o}$ represent the curvature radius coefficients of inner and outer raceways, respectively; $D_{b}$ is the diameter of ball; $\alpha_{0}$ is the initial contact angle of the bearing; $\Delta_{a}$ and $\Delta_{r}$ denote the total relative axial and radial displacements in the bearing, respectively; and $u_{c}$ represents the centrifugal displacement of the inner raceway, which has been calculated in Ref. [7].

For diverse configurations in Fig. 8, $\Delta_{a}$ is determined using different methods, and it can be described as

$$
\Delta_{a}=\left\{\begin{array}{c}
\delta_{z}+R_{i} \theta_{x} \cos \psi_{j}-R_{i} \theta_{y} \sin \psi_{j}+u_{a} \\
-\delta_{z}-R_{i} \theta_{x} \cos \psi_{j}+R_{i} \theta_{y} \sin \psi_{j}+u_{a}
\end{array}\right.
$$

(a)

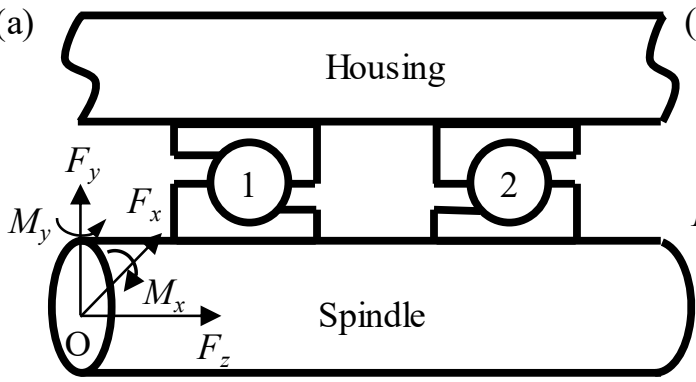

(b)

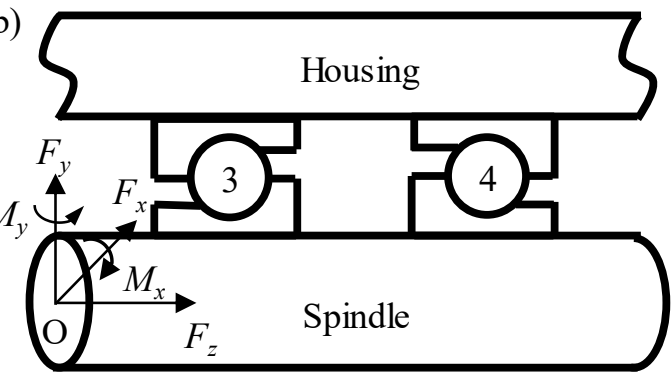

Fig. 8 Install types of bearings: (a) O-type and (b) X-type

As shown in Fig. 8, $\Delta_{a}$ in bearings 1 and 4 is determined using Eq. (26a), and that in bearings 2 and 3 is calculated using Eq. (26b). In Eq. (26), $u_{a}$ denotes the axial thermal displacement, which can be written as

$$
u_{a}=\left\{\begin{array}{ll}
u_{h}-u_{s}-\beta_{b} \Delta T_{b} D_{b}\left(\sin \alpha_{i}+\sin \alpha_{o}\right) & (27 a) \\
u_{s}-u_{h}-\beta_{b} \Delta T_{b} D_{b}\left(\sin \alpha_{i}+\sin \alpha_{o}\right) & (27 b)
\end{array},\right.
$$


where $\delta_{z}$ denotes the relative axial displacement caused by the bearing preload; $R_{i}$ represents the radius of curvature in the bearing inner raceway; $\theta_{x}$ and $\theta_{y}$ are the relative angular displacement of the bearing in $x$ and $y$ directions, respectively; $\Psi_{j}$ denotes the position angle of the ball; $u_{a}$ in bearings 1 and 2 is determined using Eq. (27a), and that in bearings 3 and 4 is calculated using Eq. (27b). In Eq. (27), $\beta_{b}$ represents the thermal conductivity of balls; $\Delta T_{b}$ denotes the temperature rise of balls; $\alpha_{i}$ is the contact angle between the ball and inner raceway; $\alpha_{o}$ is the contact angle between the ball and outer raceway under operation; $u_{h}$ and $u_{s}$ denote the thermal displacement of the bearing housing and spindle, respectively.

For the bearing housing and spindle without internal heat source, $u_{h}$ and $u_{s}$ can be expressed as follows:

$$
u(x)=\frac{c_{3} \gamma}{2} x^{2}+c_{4} \gamma x+c_{1} x+c_{2},
$$

where $\gamma$ denotes the thermal expansion coefficient of components; and $c_{1}, c_{2}, c_{3}$, and $c_{4}$ are the integral constants. $c_{3}$ and $c_{4}$ are determined using temperature boundary conditions, while $c_{1}$ and $c_{2}$ can be obtained using displacement boundary conditions.

In Eq. (25), the total relative radial displacement $\Delta_{r}$ is described as

$$
\Delta_{r}=\sqrt{\delta_{x}^{2}+\delta_{y}^{2}} \cos \left(\psi_{j}-\theta_{r}\right)+u_{r},
$$

where $\delta_{x}$ and $\delta_{y}$ are the relative displacement caused by the preload in $x$ and $y$ directions, respectively; $\theta_{r}$ denotes the angle between the ball with the maximum deformation and that with the position angle of $0^{\circ}$, which is expressed as $\theta_{r}=\arccos \left(\delta_{y} /\left(\delta_{x}{ }^{2}+\delta_{y}{ }^{2}\right)^{0.5}\right)$; and $u_{r}$ represents the radial thermal displacement in bearing and is expressed as

$$
u_{r}=\gamma_{i} \Delta T_{i} d_{i}+\left[\gamma_{s} \Delta T_{s}\left(1+\mu_{s}\right)-\gamma_{i} \Delta T_{i}\right] \frac{d^{2}}{d_{i}}-\gamma_{h} \Delta T_{h}\left(1+\mu_{h}\right) D_{o}-\gamma_{b} \Delta T_{b} D_{b}\left(\cos \alpha_{i}+\cos \alpha_{o}\right),
$$

where $\gamma_{i}, \gamma_{s}$, and $\gamma_{h}$ denote the thermal expansion coefficient of inner ring, spindle and bearing housing, respectively; $\Delta T_{i}, \Delta T_{s}$, and $\Delta T_{h}$ represent the temperature variation of inner ring, spindle, and bearing housing, respectively; $\mu_{s}$ and $\mu_{h}$ are the Poisson's ratios of spindle and bearing housing, respectively; $d$ denotes the bearing inner diameter; $d_{i}$ and $D_{o}$ are the diameters of bearing inner and outer raceways, respectively.

From Fig. 7, the geometrical compatibility equation in bearing can be written as

$$
\begin{gathered}
\left(A_{1 j}-X_{1 j}\right)^{2}+\left(A_{2 j}-X_{2 j}\right)^{2}-\left[\left(f_{i}-0.5\right) D_{b}+\delta_{i j}\right]^{2}=0, \\
X_{1 j}{ }^{2}+X_{2 j}{ }^{2}-\left[\left(f_{o}-0.5\right) D_{b}+\delta_{o j}\right]^{2}=0,
\end{gathered}
$$

where $X_{1 j}$ and $X_{2 j}$ denote the axial and radial distances between the ball and curvature centers of the outer raceway, respectively; and $\delta_{i j}$ and $\delta_{o j}$ are the contact deformation values of the inner and outer raceways contacting with the ball, respectively.

As shown in Fig. 9, force is loaded on the ball. According to outer raceway control theory, the gyroscopic moment on the ball is prevented by the contact friction between the ball and the outer raceway at high speed; otherwise, that is equally shared by the friction in the contact area.

On the basis of Hertz point contact, the force balance for the ball can be described as

$$
\begin{gathered}
K_{i j} \delta_{i j}^{1.5} \sin \alpha_{i j}-K_{o j} \delta_{o j}^{1.5} \sin \alpha_{o j}-\frac{2 M_{g j}}{D_{b}} \cos \alpha_{o j}=0, \\
K_{i j} \delta_{i j}^{1.5} \cos \alpha_{i j}-K_{o j} \delta_{o j}^{1.5} \cos \alpha_{o j}+\frac{2 M_{g j}}{D_{b}} \sin \alpha_{o j}+F_{c j}=0,
\end{gathered}
$$

where $K_{i j}$ and $K_{o j}$ denote the load deformation coefficients in the bearing; $\alpha_{i j}$ and $\alpha_{o j}$ are the contact angles between ball $j$ and the inner raceway or outer raceway, respectively; $M_{g j}$ and $F_{c j}$ represent the gyroscopic moment and centrifugal force on ball $j$, respectively. 


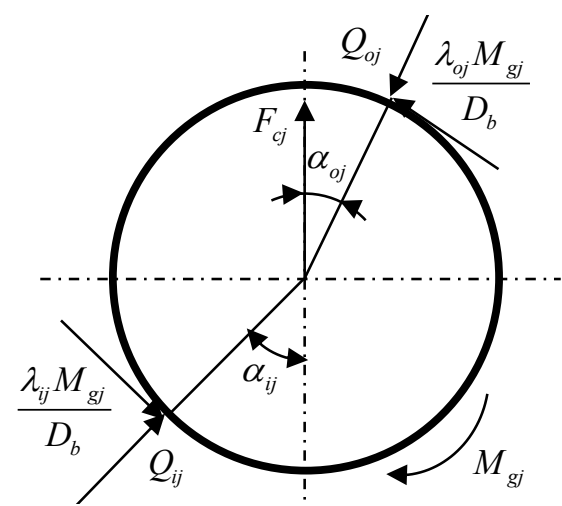

Fig. 9 Force diagram of the ball at the position angle $\Psi_{j}$

Under the equilibrium, the force balance of the bearing can be expressed as

$$
\begin{gathered}
F_{x}-\sum_{j=1}^{Z} K_{i j} \delta_{i j}^{1.5} \cos \alpha_{i j} \sin \psi_{j}=0, \\
F_{y}-\sum_{j=1}^{Z} K_{i j} \delta_{i j}^{1.5} \cos \alpha_{i j} \cos \psi_{j}=0, \\
F_{z} \mp \sum_{j=1}^{Z} K_{i j} \delta_{i j}^{1.5} \sin \alpha_{i j}=0, \\
M_{x} \mp \sum_{j=1}^{Z} R_{i} K_{i j} \delta_{i j}^{1.5} \sin \alpha_{i j} \cos \psi_{j}=0, \\
M_{y} \pm \sum_{j=1}^{Z} R_{i} K_{i j} \delta_{i j}^{1.5} \sin \alpha_{i j} \sin \psi_{j}=0,
\end{gathered}
$$

where $F_{x}, F_{y}$, and $F_{z}$ denote the external loads on the bearing in the three directions, respectively; $M_{x}$ and $M_{y}$ are the external moments; $j$ is the ordinal number of balls; and $Z$ is the number of balls. In Eqs. (37-39), the upper symbols are applied to bearings 1 and 4 , and the lower ones are used for bearings 2 and 3 .

Friction moment on bearing was divided into two parts by Palmgren [4], namely, the friction moment $M_{0}$ caused by lubricant viscosity and the friction moment $M_{1}$ caused by load. Considering the variation in bearing contact angle under the action of centrifugal force, Jorgensen and Shin [7]distributed the total friction moment to the inner and outer raceways. At the position angle $\Psi_{j}$, the friction moment on the inner and outer raceways can be expressed as

$$
\begin{gathered}
M_{i j}=0.675 f_{0}\left(\eta_{0} \omega_{c j}\right)^{2 / 3} D_{b}^{3}+f_{1}\left(\frac{Q_{i j}}{Q_{i \max }}\right)^{1 / 3} Q_{i j} D_{b}, \\
M_{o j}=0.675 f_{0}\left(\eta_{0} \omega_{c j}\right)^{2 / 3} D_{b}^{3}+f_{1}\left(\frac{Q_{o j}}{Q_{o \text { max }}}\right)^{1 / 3} Q_{o j} D_{b},
\end{gathered}
$$

where $f_{0}$ and $f_{l}$ denote the coefficients related to bearing type, lubrication, and load; $\eta_{0}$ is the kinematic viscosity of lubricant; $\omega_{c j}$ represents the common angular velocity of ball $j$; and $Q_{\text {imax }}$ and $Q_{o m a x}$ denote the maximum contact stress between the ball and inner or outer raceway, where $Q_{i \max }=\left(K_{i j} \delta_{i j}{ }^{1.5}\right)_{\max }$ and $Q_{o \max }\left(K_{o j} \delta_{o j}{ }^{1.5}\right)_{\max } . Q_{i \max }$ and $Q_{o m a x}$ can be determined by simultaneously solving Eqs. (31-39).

The spin friction moment on the ball at the inner raceway is expressed as [7]

$$
M_{s i j}=\frac{3 \mu_{s i} Q_{i j} a_{i j} \sum_{i j}}{8},
$$

where $\mu_{s i}$ denotes the friction coefficient between the ball and inner raceway, $a_{i j}$ is the half width of the Hertzian contact between ball $j$ and inner raceway, and $\Sigma_{i j}$ represents the second type of complete integral for the Hertzian contact ellipse between ball $j$ and inner raceway [13].

The bearing friction heat is determined using friction moment and angular velocity, which can be described as 


$$
\begin{gathered}
H_{i j}=\omega_{c j} \cdot M_{i j}+\omega_{b j} \cdot M_{s i j}, \\
H_{o j}=\omega_{c j} \cdot M_{o j}, \\
H_{b}=\sum_{j=1}^{Z} H_{i j}+H_{i j},
\end{gathered}
$$

where $H_{i j}$ and $H_{o j}$ denote the friction heat of the contact area, $H_{b}$ represents the total friction heat of the bearing, and $\omega_{b j}$ represents the angular velocity of the ball.

In summary, to acquire the friction heat of bearings, the contact load is required by solving Eqs. (31-39). The contact load of bearings is closely related to the temperature field of the system and the thermal deformation of bearings. On the other hand, the friction heat of bearings must be gained first to predict the temperature field of the system (Eq. 3). Therefore, the friction heat of bearings and the temperature field of the system are coupled with each other and cannot be solved independently. An iterative algorithm is provided to solve the coupled equations in the next section.

\section{Numerical solution and experimental verification}

\subsection{Numerical solution}

Fig. 10 illustrates the solution flowchart of the thermal network model for the motorized spindle. First, the motor power loss $H_{m}$ and bearings friction heat $H_{b 0}$ are determined using the methods introduced in Subsection 2.3, where $H_{b 0}$ is calculated when $\Delta_{a}$ and $\Delta_{r}$ are zero. Second, the temperature field [T] of the motorized spindle can be obtained by substituting the heat into Eq. (3). Third, the thermal displacement of bearings can be acquired from the temperature field, and Eqs. (31) - (39) are solved to gain bearing friction heat $H_{b 1}$ by substituting the thermal displacement. The steady temperature field $[\mathbf{T}]$ of the motorized spindle is output until the convergence criterion is satisfied, where, the convergence criterion can be given as

$$
\left|\frac{H_{b 1}-H_{b 0}}{H_{b 0}}\right| \leq \varepsilon .
$$

Otherwise, $H_{b 0}=H_{b 1}$ is assigned, and Eq. (3) is resolved. $\varepsilon$ is the user-defined error threshold, and $\varepsilon=(10)^{-4}$ is found to be exact for the example analysis presented in the next section by repeatedly comparing of simulated results.

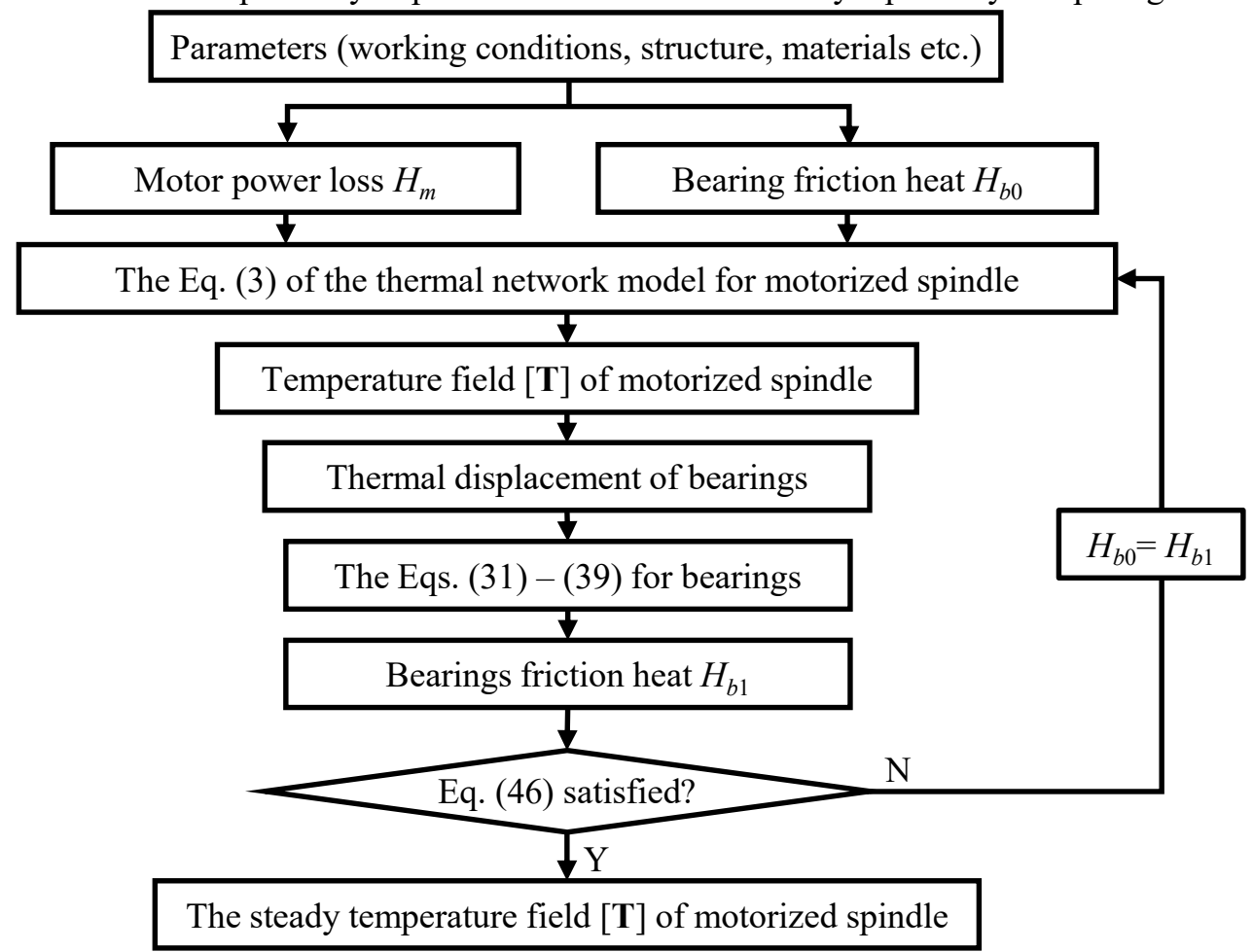

Fig. 10 Computation flowchart of the thermal network model 


\subsection{Experimental verification}

(a)

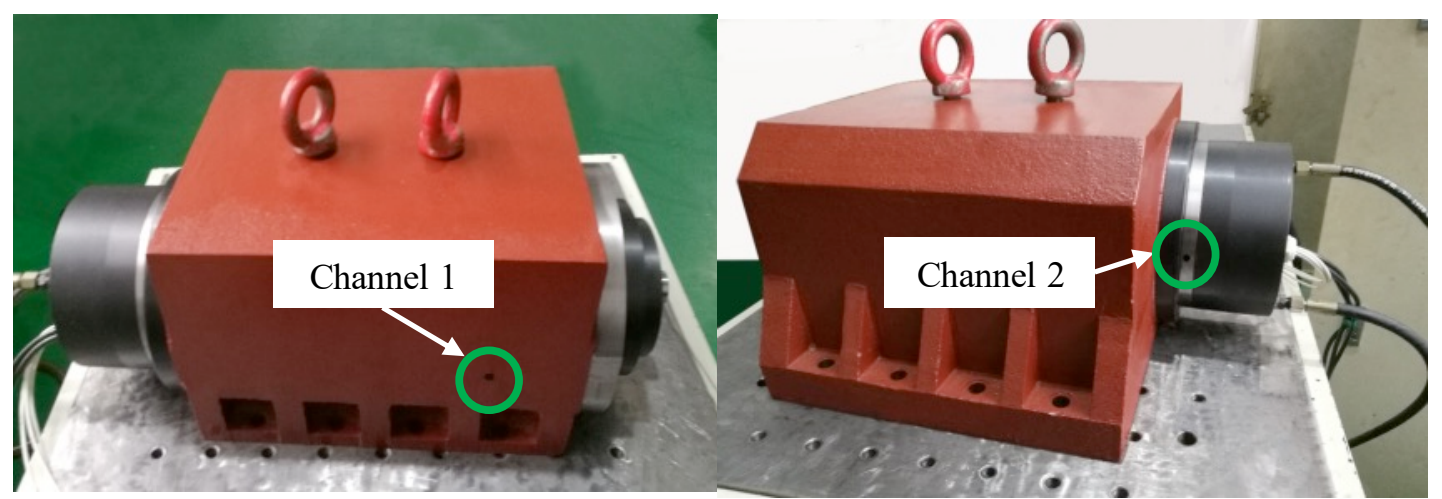

(b)

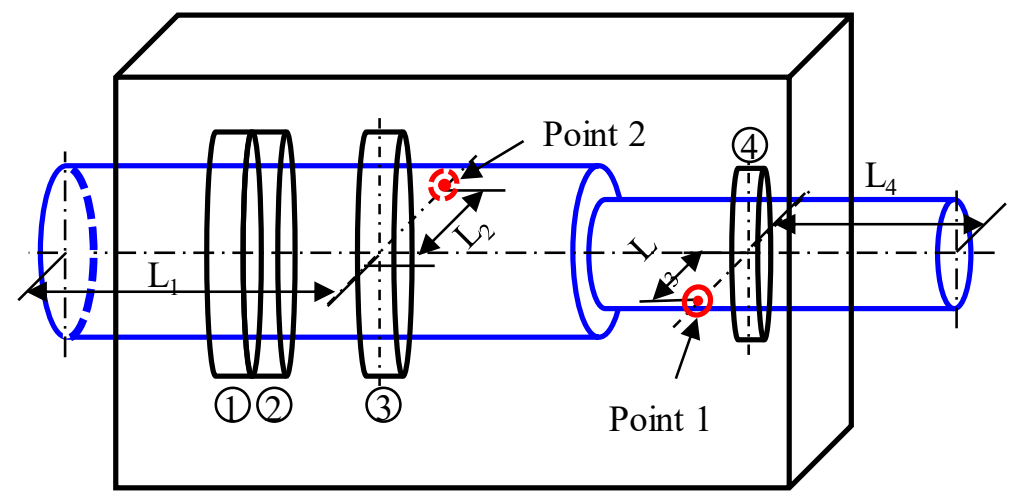

Fig. 11 Temperature test rig and measuring positions

The temperatures near the outer ring of bearings 3 and 4 are tested using a thermocouple sensor to verify the thermal network model (Fig. 11). The test rig is shown in Fig. 11a, and the position dimensions of the measuring points are $L_{1}=212.5 \mathrm{~mm}, L_{2}=80 \mathrm{~mm}, L_{3}=61.25 \mathrm{~mm}$, and $L_{4}=63 \mathrm{~mm}$ (Fig. 11b). In this work, the spindle runs for more than $3 \mathrm{~h}$ to ensure that the spindle is in thermal balance, then the temperature value on the temperature indicator can be recorded.

Table 1 Basic parameters of bearings

\begin{tabular}{lcc}
\hline \multicolumn{1}{c}{ Parameters } & Bearing 3 (B7018C) & Bearing 4 (B7011C) \\
\hline Medium diameter of bearing/mm & 115 & 72.5 \\
Diameter of balls/mm & 13.75 & 9.27 \\
Curvature radius coefficient of the inner raceway & 0.57 & 0.57 \\
Curvature radius coefficient of the outer raceway & 0.54 & 0.54 \\
Initial contact angle ${ }^{\circ}$ & 15 & 15 \\
Number of balls & 25 & 20 \\
\hline
\end{tabular}

Table 2 Materials of parts in the motorized spindle system

\begin{tabular}{ccccccc}
\hline Parts & Spindle & Bearing ball & Bearing ring & Stator & Rotor & Bearing housing \\
\hline Material & 38CrMoAlA & Si3N4 & GCr15 & Silicon steel & Nd2Fe14B & $40 \mathrm{Cr}$ \\
\hline
\end{tabular}

In this test, the rated speed of the motorized spindle is $3000 \mathrm{rpm}$, and the ambient temperature is $26^{\circ} \mathrm{C}$. The cooling oil volume flow rate of the stator is $4 \mathrm{~L} / \mathrm{min}$, and the inlet oil temperature is $21^{\circ} \mathrm{C}$. All bearings are preloaded by positioning, and the initial preload is $150 \mathrm{~N}$. The bearing parameters and materials of the components are shown in Tables 1 and 2, respectively. 

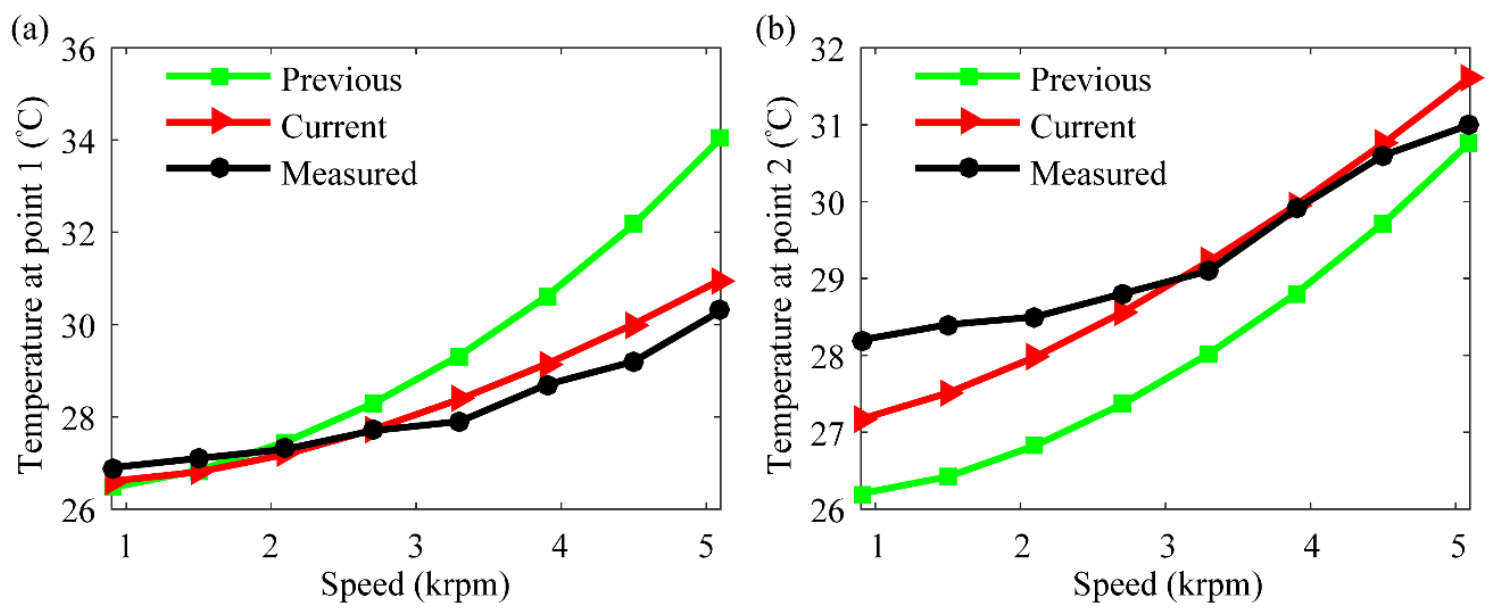

Fig. 12 The predicted and measured temperature at: (a) point 1 and (b) point 2

Table 3 The measured and predicted temperature

\begin{tabular}{ccccccc}
\hline \multirow{2}{*}{ Speed (rpm) } & \multicolumn{3}{c}{ Temperature at Point $1\left({ }^{\circ} \mathrm{C}\right)$} & \multicolumn{3}{c}{ Temperature at Point $2\left({ }^{\circ} \mathrm{C}\right)$} \\
\cline { 2 - 6 } & Measured & Previous & Current & Measured & Previous & Current \\
\hline 900 & 26.9 & 26.5 & 26.6 & 28.2 & 26.2 & 27.2 \\
1500 & 27.1 & 26.8 & 26.8 & 28.4 & 26.4 & 27.5 \\
2100 & 27.3 & 27.3 & 27.2 & 28.5 & 26.8 & 28.0 \\
2700 & 27.7 & 28.0 & 27.7 & 28.8 & 27.3 & 28.6 \\
3300 & 27.9 & 28.8 & 28.4 & 29.1 & 27.8 & 29.3 \\
3900 & 28.7 & 29.8 & 29.1 & 29.9 & 28.4 & 30.0 \\
4500 & 29.2 & 30.9 & 30.0 & 30.6 & 29.1 & 30.8 \\
5100 & 30.3 & 32.2 & 31.0 & 31.0 & 29.9 & 31.6 \\
MRE (\%) & $/$ & 12.33 & 2.77 & $/$ & 7.09 & 3.65 \\
\hline
\end{tabular}

The measured and predicted temperatures near the outer ring of bearings 3 and 4 are illustrated in Fig. 12 and Table 3. The current results are calculated using the proposed model, whereas the previous results refer to the calculated result without the thermal-mechanical coupling effect. The friction heat becomes large with the increase in rotation speed, thereby causing a high temperature near the outer ring. For the previous result, the maximum relative errors (MREs) of temperature near bearings 3 and 4 are 12.33\% and 7.09, respectively. By contrast, the MREs for the current result decrease to $2.77 \%$ and $3.65 \%$. The comparison results indicate that the predicted temperature including thermal-mechanical coupling effect is consistent with the measured temperature. After the thermal-mechanical coupling effect is considered, the temperature rise is affected by the change in dynamic performance, such as bearing displacement, contact angle, contact load, and friction heat. The reasons for the difference between the previous and current results will be elucidated in the next section.

\section{Results and discussions}

Other parameters of the motorized spindle need to be qualitatively analyzed to understand the reasons for the difference between the previous and current results. In consideration of the position of measured points, the thermal parameters of bearings 3 and 4 are analyzed in this section. 

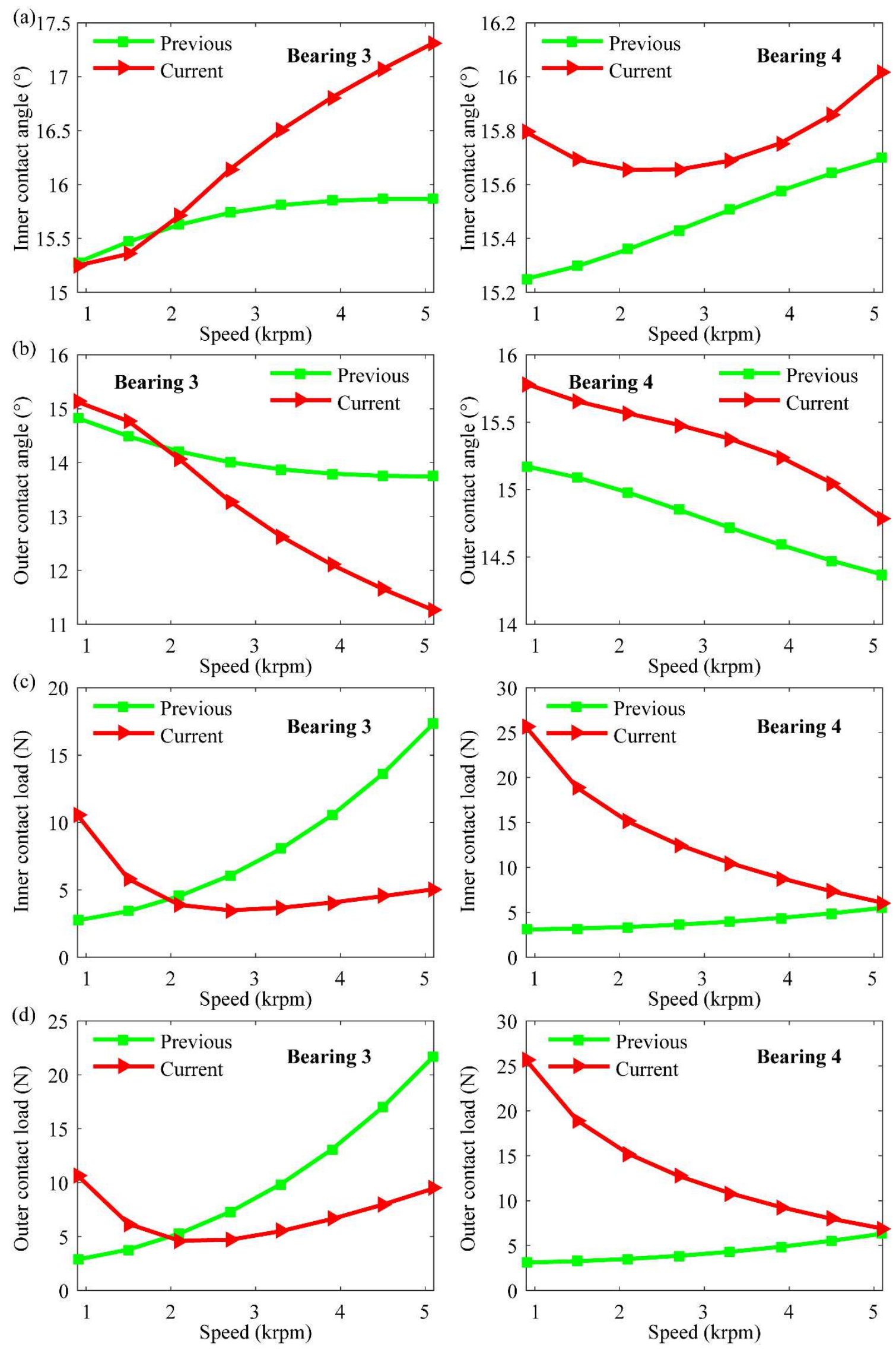

Fig. 13 Contact angle and contact load of inner and outer rings

Fig. 13 shows the contact angle and load of bearings 3 and 4 . The change law of the contact angle and load of bearing 3 predicted using the current model is the same as that of bearing 4 . However, a difference exists between the current results of bearings 3 and 4 . For bearing 3, the centrifugal force of balls becomes large with the increase 
in rotation speeds, thereby increasing the inner contact angle and decreasing the outer contact angle. The outer contact angle of bearing 4 also decreases with the increase in speeds, whereas its inner contact angle first decreases and then increases. The diameter of balls of bearing 4 is smaller than that of bearing 3 ; thus, the centrifugal force of balls of bearing 4 is not dominant at low speeds. On the contrary, the axial thermal displacement of bearing 4 changes sharply at low speeds (Fig. 15 in Appendix), thereby causing a reduction in the inner contact angle in the range of 900-2100 rpm. Likewise, the contact load of bearing 3 decreases at low speeds, which is mainly caused by the axial thermal displacement. With the increase in rotation speeds, the centrifugal force and radial thermal displacement play dominant roles, thereby increasing the inner and outer contact loads of bearing 3 . The influence of centrifugal force is insufficient to reverse the influence of thermal displacement on contact loads in the range of 900-5100 rpm. Therefore, the inner and outer contact loads of bearing 4 decrease with the increase in rotation speeds.

Fig. 13 indicates that the contact angle and load obtained using the two methods are relatively different. The contact angle and load of bearings are affected by thermal deformation, thereby changing the frictional heat, as shown in Fig. 14. The comparison analysis between the current and previous results shows that the temperature rise and thermal deformation have considerable effects on the contact performance of bearings, and the change in the contact performance will in turn affect the temperature rise and thermal deformation of the system. These analysis results explain why the current result is more accurate than the previous one.
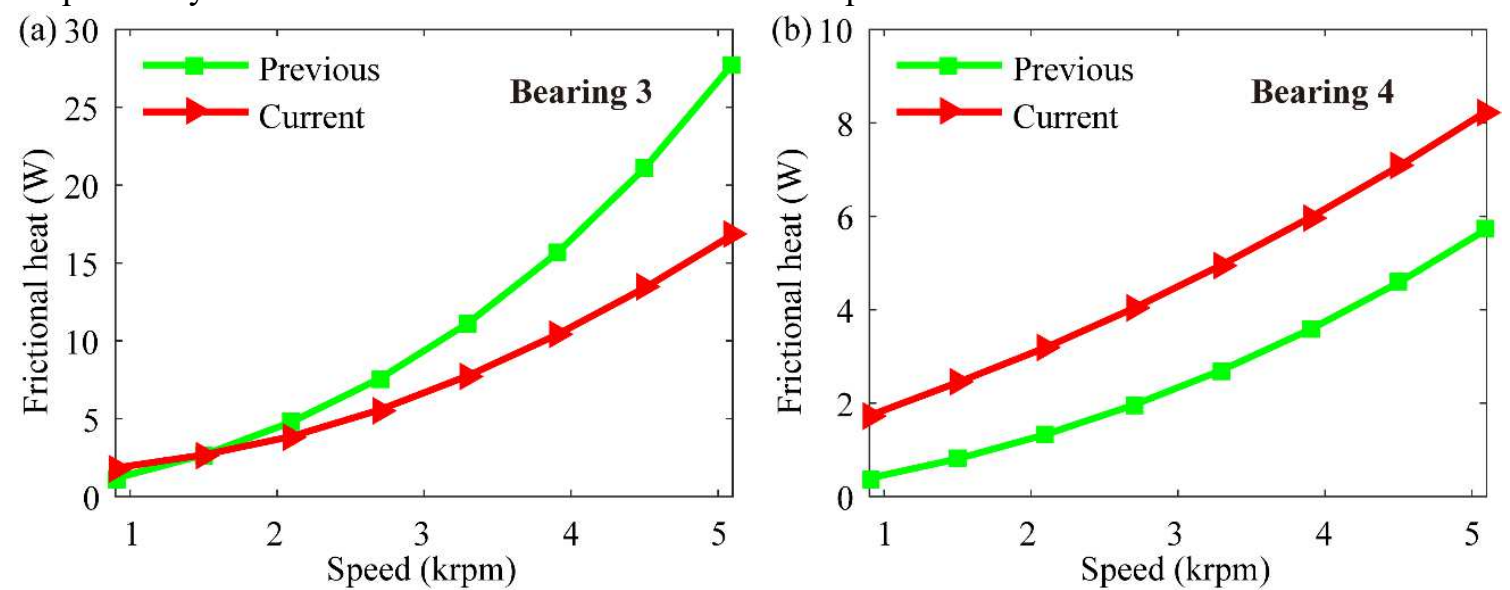

Fig. 14 Frictional heat of bearings

\section{Conclusions}

An enhanced thermal network model of motorized spindle was proposed by considering the thermalmechanical coupling effect. In accordance with this model, we obtained the temperature field of the motorized spindle and analyzed the temperature near bearings at different rotation speeds. Then, the proposed model was verified via temperature experiment. The conclusions are summarized as follows:

(1) The temperature predicted by the proposed model is more accurate than that predicted by the previous model (without thermal-mechanical coupling effect). The measured temperature is regarded as a baseline. The MREs of temperature near bearings 3 and 4 decrease by $9.56 \%$ and $3.44 \%$, respectively, through considering the thermal-mechanical coupling effect. The comparison result shows that the thermal-mechanical coupling effect needs to be included to predict the temperature field of motorized spindle systems.

(2) Temperature changes the contact performance of bearings (contact angle, contact load, etc.), thereby causing changes in frictional heat and temperature field. Such changes cause the difference between the current and previous results.

Compared with the previous model, the enhanced thermal network model can obtain more accurate temperature. Therefore, the proposed model can be efficiently applied to investigate the thermal performance of motorized spindles, and the analysis results are valuable to the design and improvement of motorized spindle systems.

\section{Declarations}

Funding: This work was supported by the National Natural Science Foundation of China (Grants Nos. 52005051 
and 52075153) and the Key Research and Development Program of Hunan Province (Grants Nos. 2020WK2032 and 2016JC2001).

Conflicts of interest: The authors have no conflicts of interest to declare that are relevant to the content of this article.

Availability of data and material: Not applicable.

Code availability: Not applicable.

Authors' contributions: Zhou C.: Methodology, Formal analysis, Investigation, Resources, Data curation, Writing - review \& editing, Visualization, Project administration, Funding acquisition. Qu Z.: Methodology, Software, Investigation, Writing - original draft. Hu B.: Conceptualization, Methodology, Validation, Investigation, Resources, Writing - review \& editing, Supervision, Project administration, Funding acquisition. Li S.: Conceptualization, Methodology, Writing - review \& editing.

Ethics approval: Not applicable.

Consent to participate: Not applicable.

Consent for publication: Not applicable.

Appendix
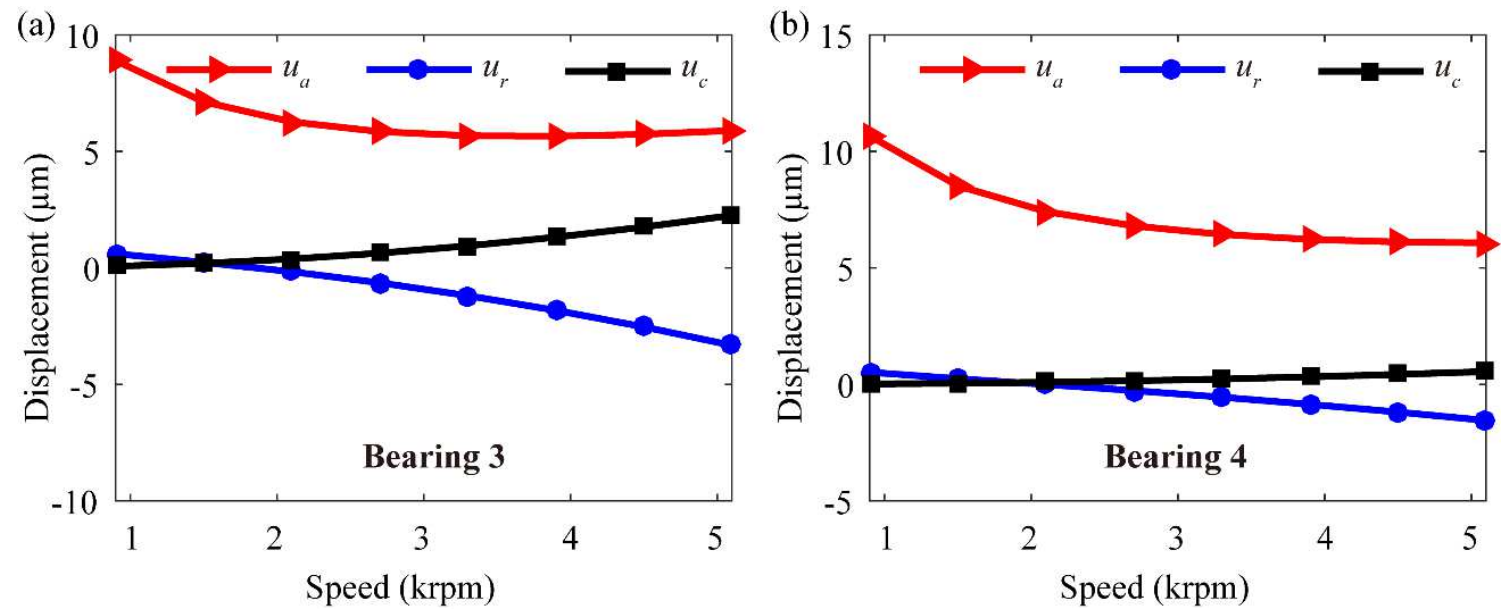

Fig. 15 Thermal displacement and centrifugal displacement of bearings 3 and 4

\section{References}

1. Abele E, Altintas Y, Brecher C (2010) Machine tool spindle units. CIRP Annals - Manufacturing Technology 59 (2):781-802. https://doi.org/10.1016/j.cirp.2010.05.002

2. Hu B, Zhou C, Wang H, Yin L (2021) Prediction and validation of dynamic characteristics of a valve train system with flexible components and gyroscopic effect. Mechanism and Machine Theory 157:104222. https://doi.org/10.1016/j.mechmachtheory.2020.104222

3. Shi X, Yang X, Mu Y, Wang Y, Wang W (2019) Thermal error compensation model for a motorized spindle with shaft core cooling based on exponential function. The International Journal of Advanced Manufacturing Technology 103 (9):4805-4813. https://doi.org/10.1007/s00170-019-04038-w

4. Palmgren A (1959) Ball and roller bearing engineering. Philadelphia: SKF Industries Inc., 1959.

5. Jones AB (1959) Ball Motion and Sliding Friction in Ball Bearings. Journal of Basic Engineering 81 (1):1-12. https://doi.org/10.1115/1.4008346

6. Harris TA, Mindel MH (1973) Rolling element bearing dynamics. Wear 23 (3):311-337. https://doi.org/10.1016/0043-1648(73)90020-3

7. Jorgensen BR, Shin YC (1997) Dynamics of machine tool spindle/bearing systems under thermal growth. Journal of Tribology 119 (4):875-882. https://doi.org/10.1115/1.2833899 
8. Donmez MA, Hahn MH, Soons JA (2007) A Novel Cooling System to Reduce Thermally-Induced Errors of Machine Tools. CIRP Annals - Manufacturing Technology 56 (1):521-524. https://doi.org/10.1016/j.cirp.2007.05.124

9. Zhao H, Yang J, Shen J (2007) Simulation of thermal behavior of a CNC machine tool spindle. International Journal of Machine Tools \& Manufacture 47 (6):1003-1010. https://doi.org/10.1016/j.ijmachtools.2006.06.018

10. Jiang S, Min X (2012) Thermal design of the vertical machining centre headstock by the forced cooling method. Proceedings of the Institution of Mechanical Engineers, Part C: Journal of Mechanical Engineering Science 226 (3):738-751. https://doi.org/10.1177/0954406211414642

11. Bossmanns B, Tu JF (1999) A thermal model for high speed motorized spindles. International Journal of Machine Tools and Manufacture 39 (9):1345-1366. https://doi.org/10.1016/S0890-6955(99)00005-X

12. Chen J-S, Hsu W-Y (2003) Characterizations and models for the thermal growth of a motorized high speed spindle. International Journal of Machine Tools and Manufacture 43 (11):1163-1170. https://doi.org/10.1016/s0890-6955(03)00103-2

13. Li H, Shin YC (2004) Integrated dynamic thermo-mechanical modeling of high speed spindles, part 1: model development. J Manuf Sci Eng 126 (1):148-158. https://doi.org/10.1115/1.1644545

14. Li H, Shin YC (2004) Integrated dynamic thermo-mechanical modeling of high speed spindles, part 2: solution procedure and validations. J Manuf Sci Eng 126 (1):159-168. https://doi.org/10.1115/1.1644546

15. Ye CXLJH, Wentao ZPS (2013) Thermal properties of high speed motorized spindle and their effects. Journal of Mechanical Engineering (11):20. https://doi.org/10.3901/JME.2013.11.135

16. Lee J, Kim D-H, Lee C-M (2015) A study on the thermal characteristics and experiments of high-speed spindle for machine tools. International Journal of Precision Engineering and Manufacturing 16 (2):293-299. https://doi.org/10.1007/s12541-015-0039-8

17. Zivkovic A, Zeljkovic M, Tabakovic S, Milojevic Z (2015) Mathematical modeling and experimental testing of high-speed spindle behavior. The International Journal of Advanced Manufacturing Technology 77 (5-8):10711086. https://doi.org/10.1007/s00170-014-6519-7

18. Than VT, Wang CC, Ngo TT, Huang JH (2017) Estimating time-varying heat sources in a high speed spindle based on two measurement temperatures. International Journal of Thermal ences 111:50-65. https://doi.org/10.1016/j.ijthermalsci.2016.08.004

19. Yang A-S, Yu X-H, Zhuang J-R, Lee C-Y, Hsieh W-H (2018) DOE-FEM based design improvement to minimize thermal errors of a high speed spindle system. Thermal Science and Engineering Progress 8:525-536. https://doi.org/10.1016/j.tsep.2018.10.011

20. Liu J, Ma C, Wang S, Wang S, Yang B, Shi H (2019) Thermal-structure interaction characteristics of a highspeed spindle-bearing system. International Journal of Machine Tools and Manufacture 137:42-57. https://doi.org/10.1016/j.ijmachtools.2018.10.004

21. Zhang L, Gong W, Zhang K, Wu Y, An D, Shi H, Shi Q (2018) Thermal deformation prediction of high-speed motorized spindle based on biogeography optimization algorithm. The International Journal of Advanced Manufacturing Technology 97 (5):3141-3151. https://doi.org/10.1007/s00170-018-2123-6

22. Yan Z, Tao T, Hou R, Du H, Mei X (2020) A new modeling method for thermal errors of motorized spindle based on the variation characteristics of spindle temperature field. The International Journal of Advanced Manufacturing Technology 110 (3):989-1000. https://doi.org/10.1007/s00170-020-05752-6

23. Uhlmann E, Hu J (2012) Thermal Modelling of a High Speed Motor Spindle. Procedia Cirp 1 (1):313-318. https://doi.org/10.1016/j.procir.2012.04.056

24. Xu M, Jiang S, Cai Y (2007) An improved thermal model for machine tool bearings. International Journal of Machine Tools \& Manufacture 47 (1):53-62. https://doi.org/10.1016/j.ijmachtools.2006.02.018

25. Ma C, Mei X, Yang J, Zhao L (2015) Thermal characteristics analysis and experimental study on the high-speed spindle system. Journal of Zhejiang University 79 (1-4):469-489 
26. Ma C, Yang J, Zhao L, Mei X, Shi H (2015) Simulation and experimental study on the thermally induced deformations of high-speed spindle system. Applied Thermal Engineering 86:251-268

27. Cui Y, Li H, Li T, Chen L (2018) An accurate thermal performance modeling and simulation method for motorized spindle of machine tool based on thermal contact resistance analysis. The International Journal of Advanced Manufacturing Technology 96 (5-8):2525-2537. https://doi.org/10.1007/s00170-018-1593-x

28. Zhang L, Li C, Wu Y, Zhang K, Shi H (2017) Hybrid Prediction Model of the Temperature Field of a Motorized Spindle. Applied Sciences 7 (10):1091. https://doi.org/10.3390/app7101091

29. Hu B, Zhou C, Wang H, Chen S (2021) Nonlinear tribo-dynamic model and experimental verification of a spur gear drive under loss-of-lubrication condition. Mechanical Systems and Signal Processing 153:107509. https://doi.org/10.1016/j.ymssp.2020.107509

30. Liu X, Liu D, Hu X (2021) Influence of the Bearing Thermal Deformation on Nonlinear Dynamic Characteristics $\begin{array}{lllllll}\text { of an Electric Drive Helical Gear } & \text { System. } & \text { Sensors }\end{array}$ https://doi.org/10.1016/j.mechmachtheory.2020.104222

31. Liu Z, Pan M, Zhang A, Zhao Y, Yang Y, Ma C (2015) Thermal characteristic analysis of high-speed motorized spindle system based on thermal contact resistance and thermal-conduction resistance. The International Journal of Advanced Manufacturing Technology 76 (9):1913-1926. https://doi.org/10.1007/s00170-014-6350-1

32. Yan K, Hong J, Zhang J, Mi W, Wu W (2016) Thermal-deformation coupling in thermal network for transient analysis of spindle-bearing system. International Journal of Thermal Sciences 104:1-12. https://doi.org/10.1016/j.ijthermalsci.2015.12.007

33. Zheng D, Chen W, Zheng D (2020) An enhanced estimation on heat generation of angular contact ball bearings with vibration effect. International Journal of Thermal Sciences 159:106610

34. Liu Y, Ma Y-X, Meng Q-Y, Xin X-C, Ming S-S (2018) Improved thermal resistance network model of motorized spindle system considering temperature variation of cooling system. Advances in Manufacturing 6 (4):384-400. https://doi.org/10.1007/s40436-018-0239-4

35. Bi J, Chen X, Li Y (2014) Thermal performance analysis of high-speed high-performance motorized spindle. J Mech Transm 35:84-87

36. Bertotti G (1988) General properties of power losses in soft ferromagnetic materials. IEEE Transactions on Magnetics 24 (1):621-630. https://doi.org/10.1109/20.43994 
Figures

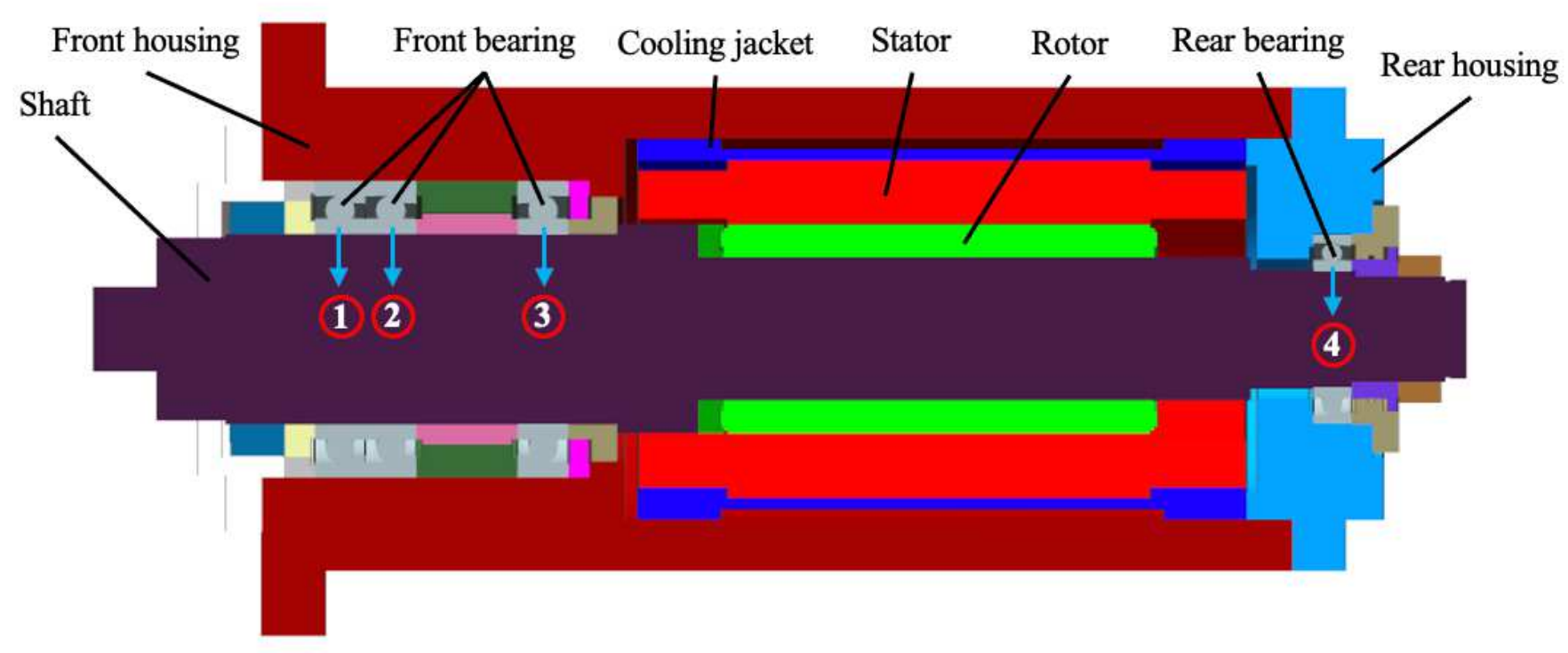

Figure 1

Geometric model of motorized spindle

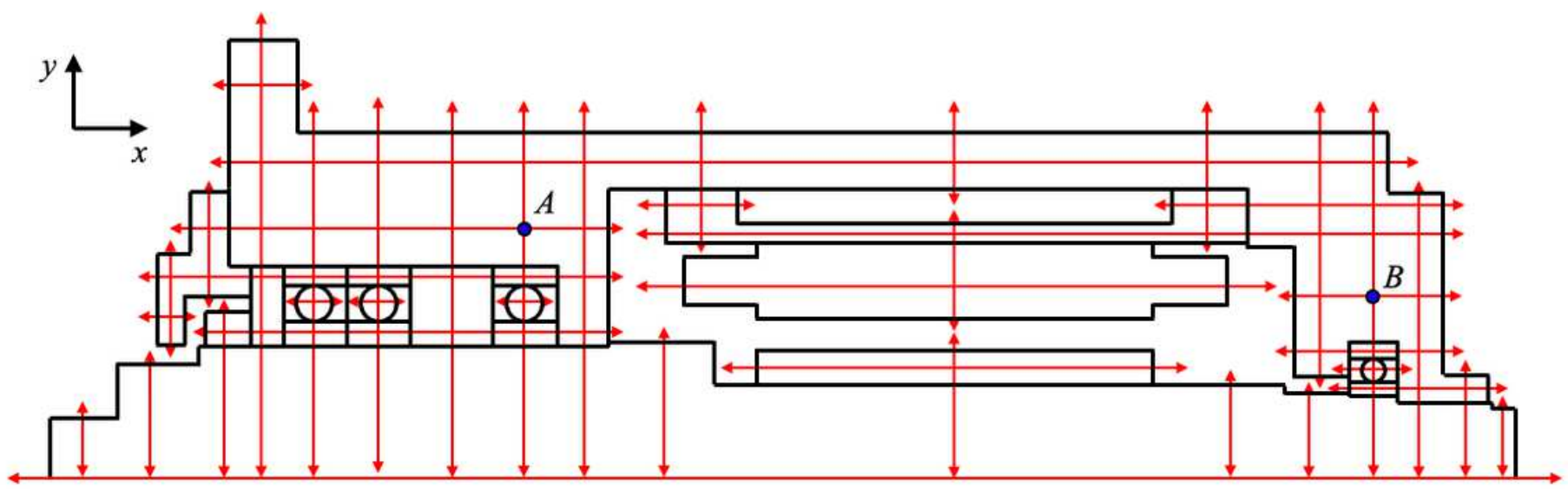

Figure 2

Heat transfer path of motorized spindle 


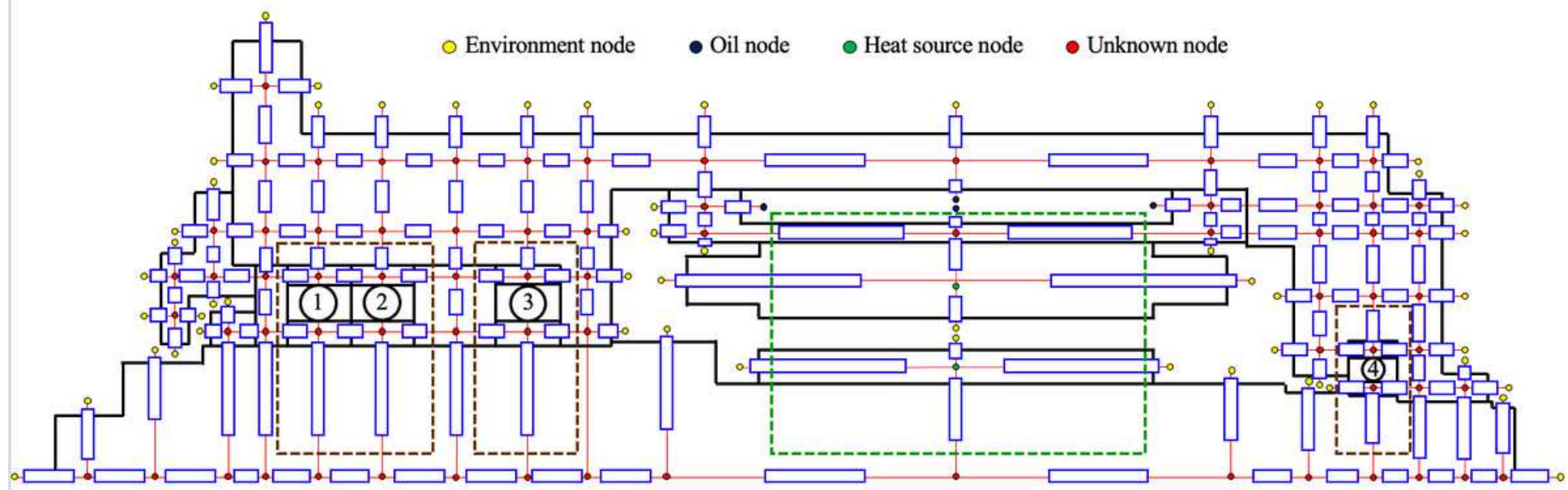

Figure 3

Global thermal network model of motorized spindle

(a)

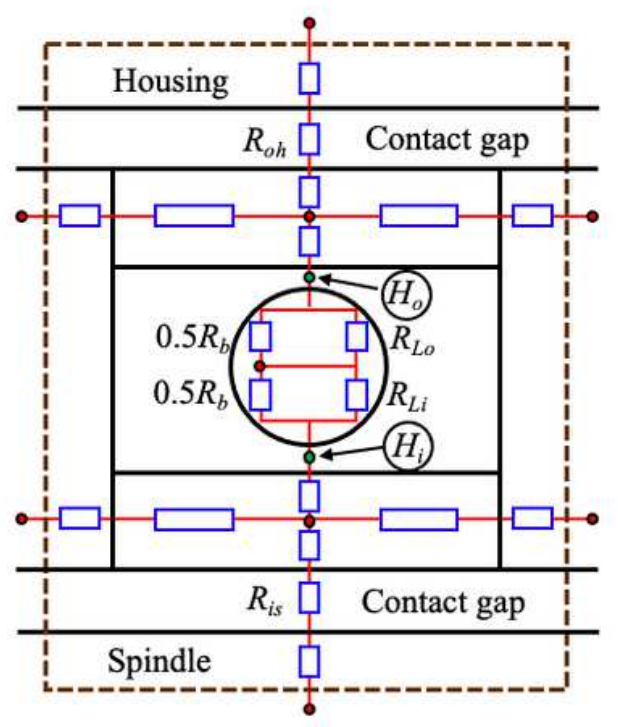

(b)

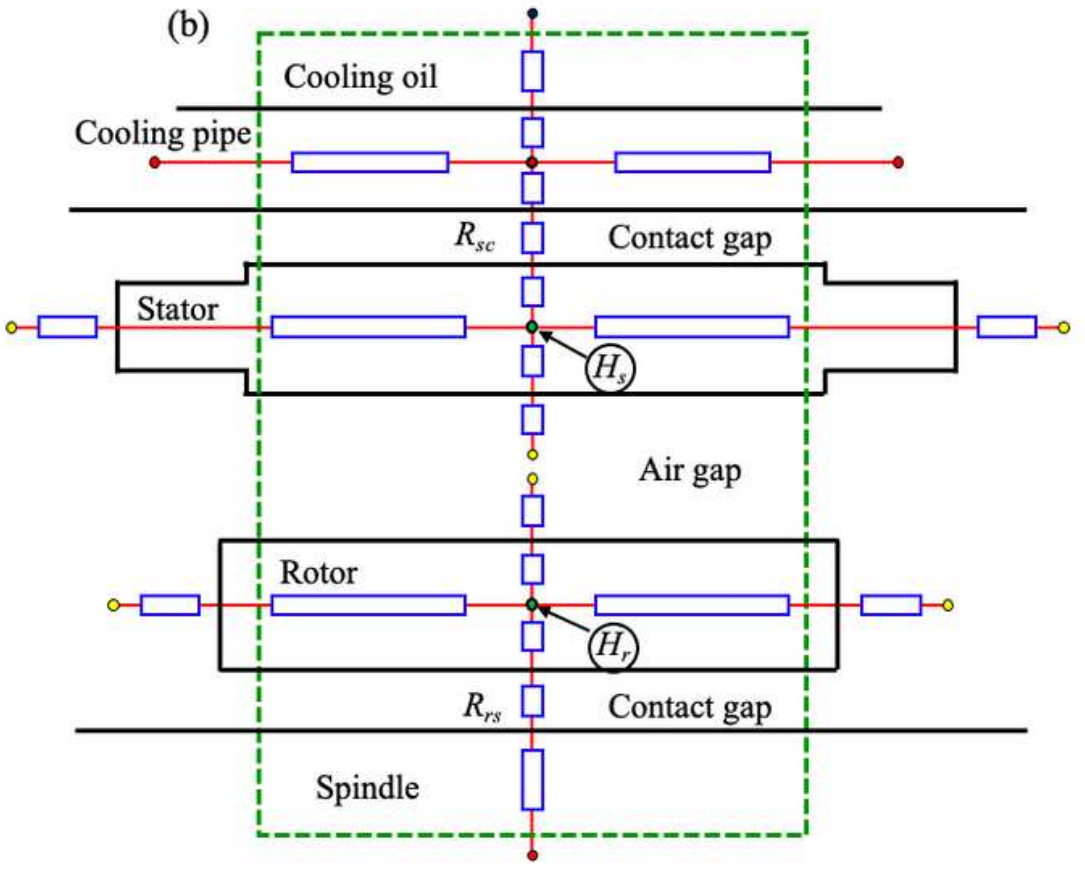

\section{Figure 4}

Local thermal network model for (a) bearings and (b) motor 


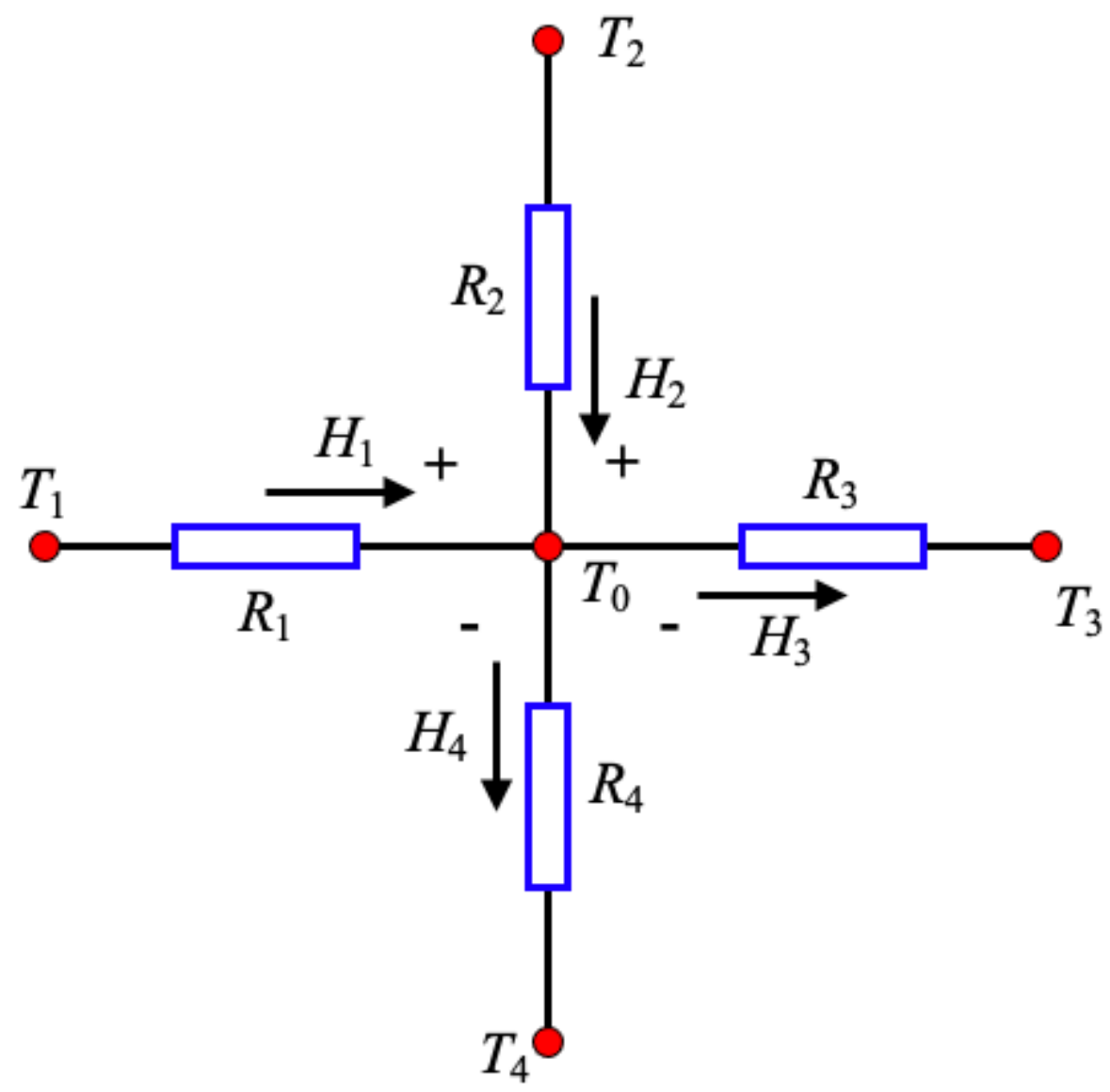

Figure 5

Heat transfer diagram 


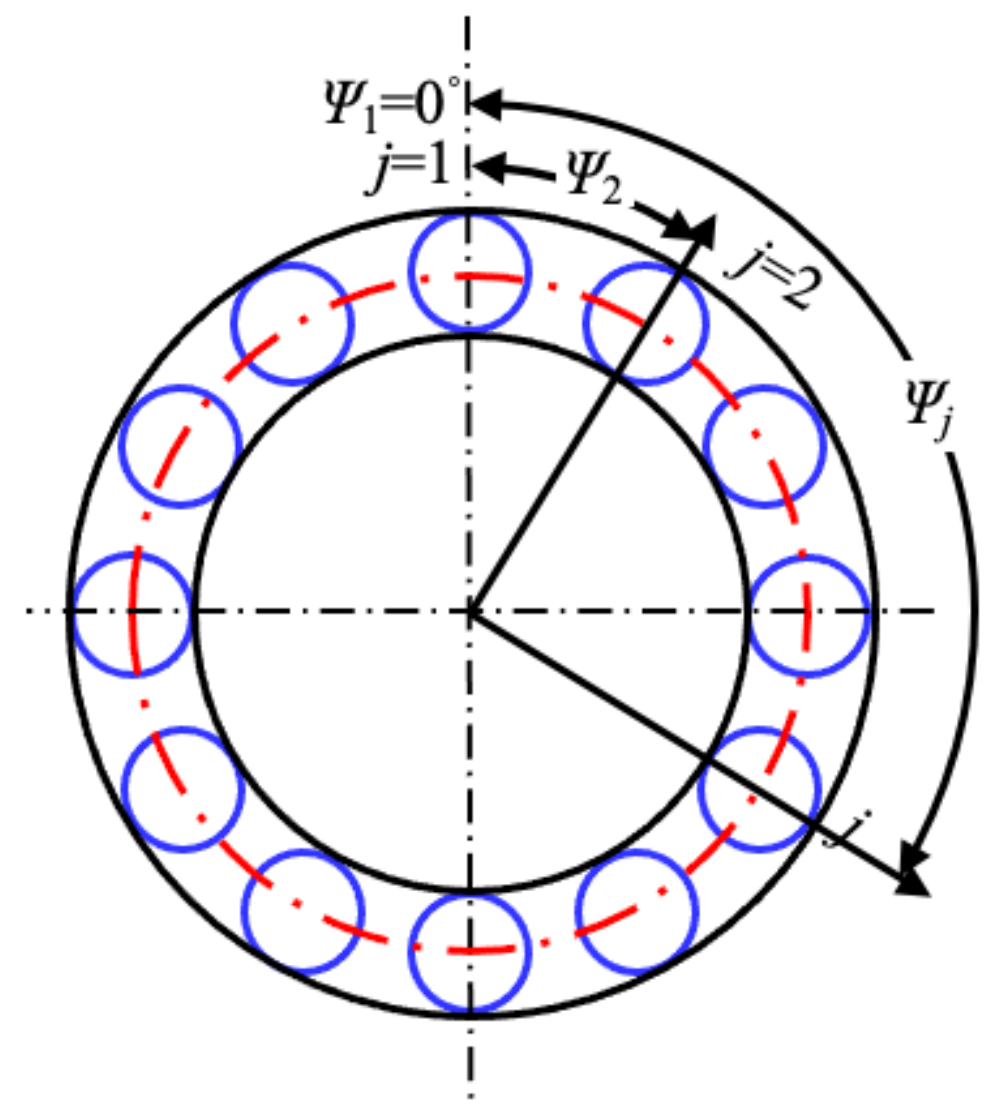

Figure 6

Position angle of balls 


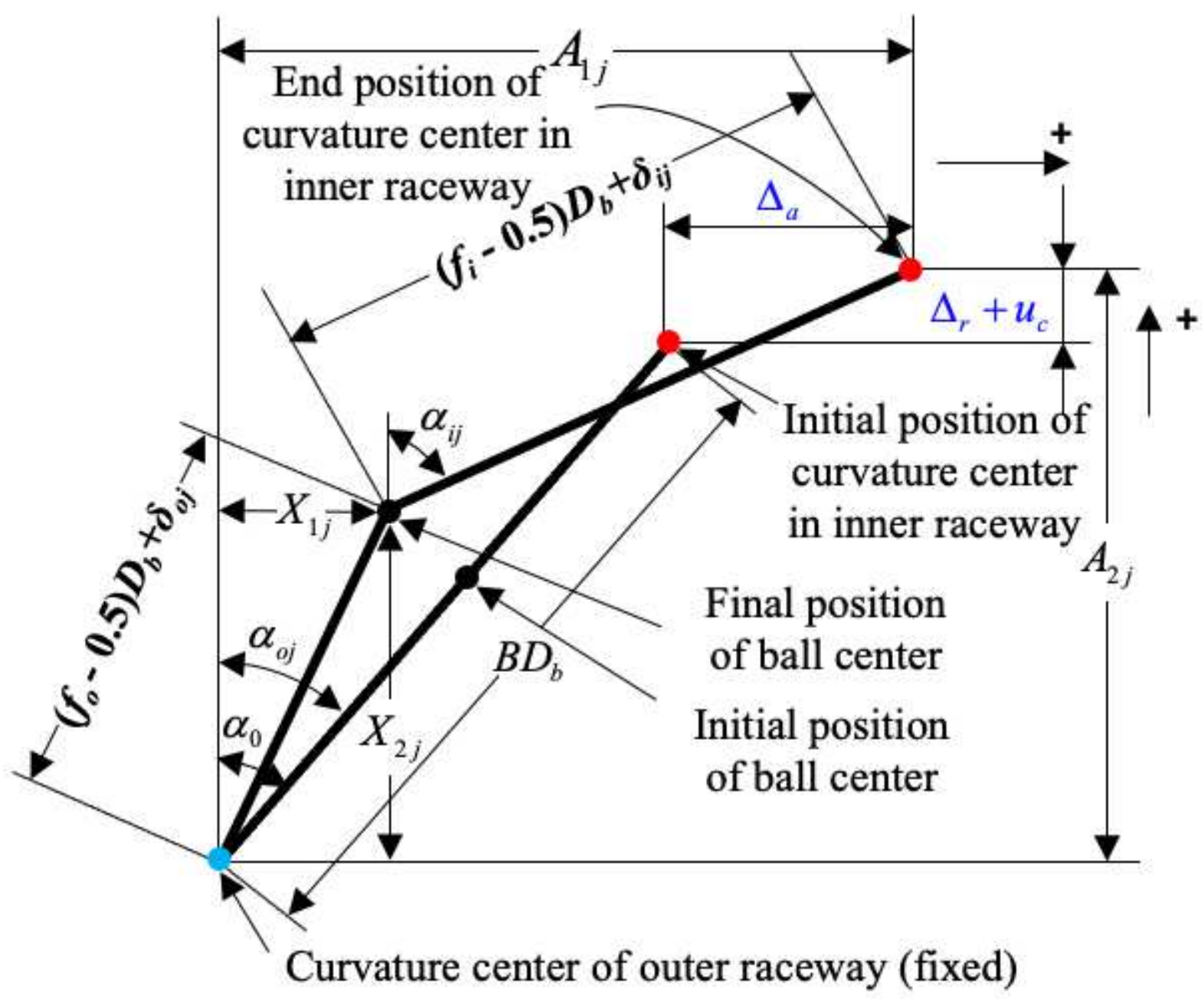

Figure 7

Position of ball, inner and outer rings before and after loading 

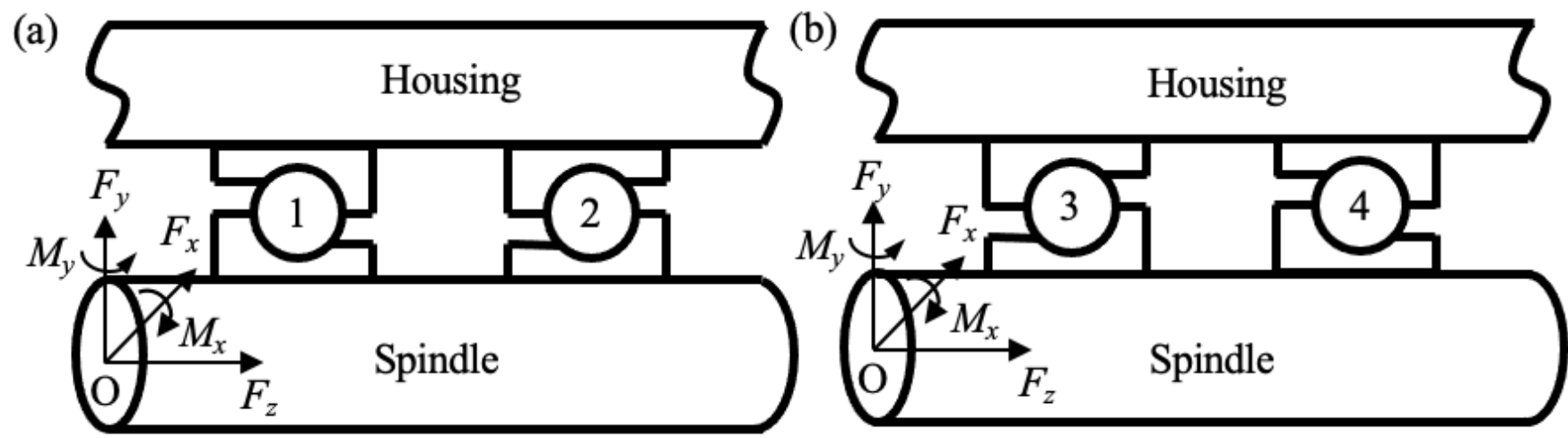

Figure 8

Install types of bearings: (a) O-type and (b) X-type

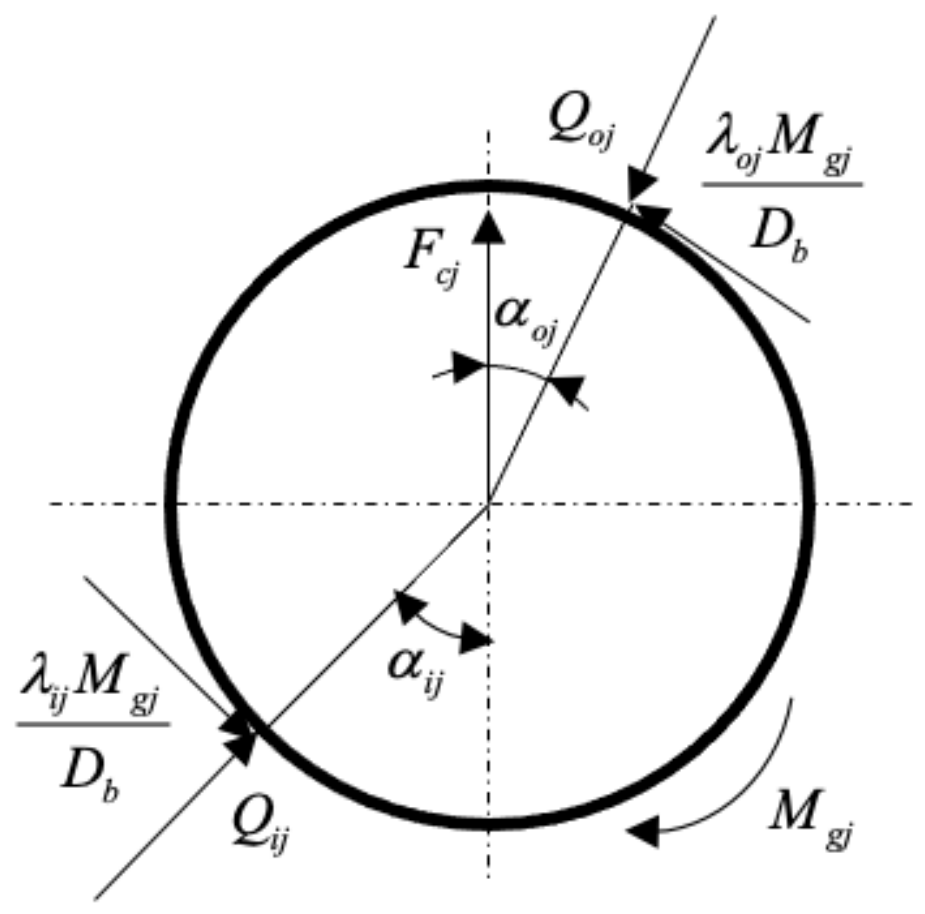

Figure 9

Force diagram of the ball at the position angle $\Psi_{\mathrm{j}}$ 


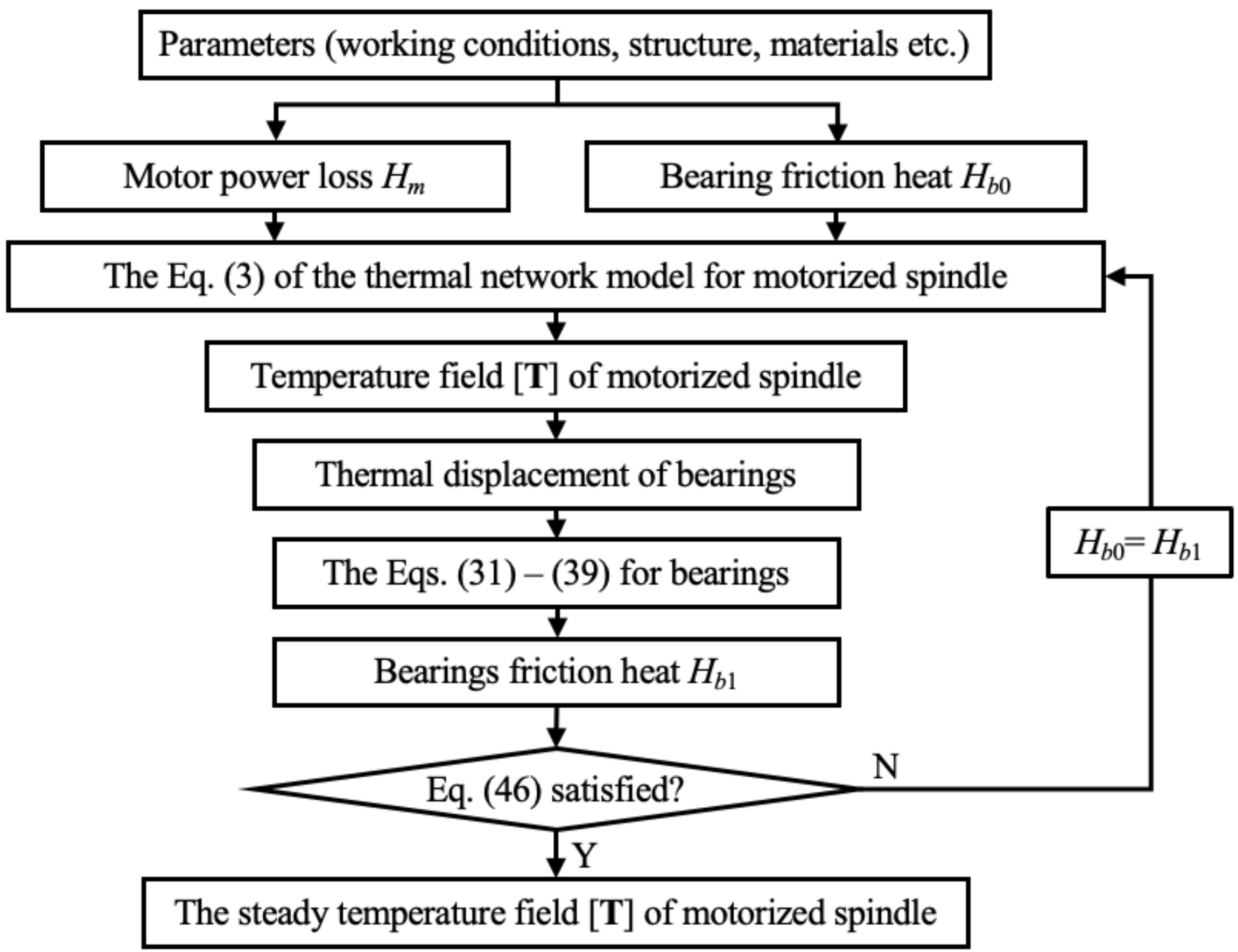

Figure 10

Computation flowchart of the thermal network model 
(a)

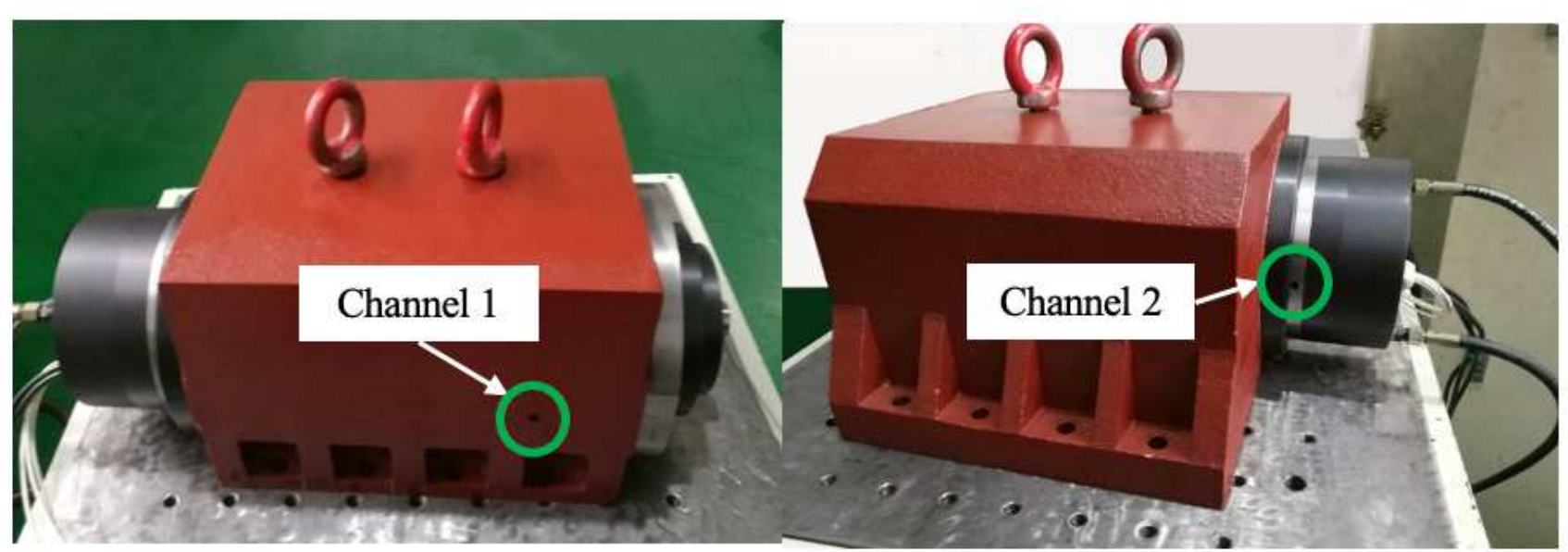

(b)

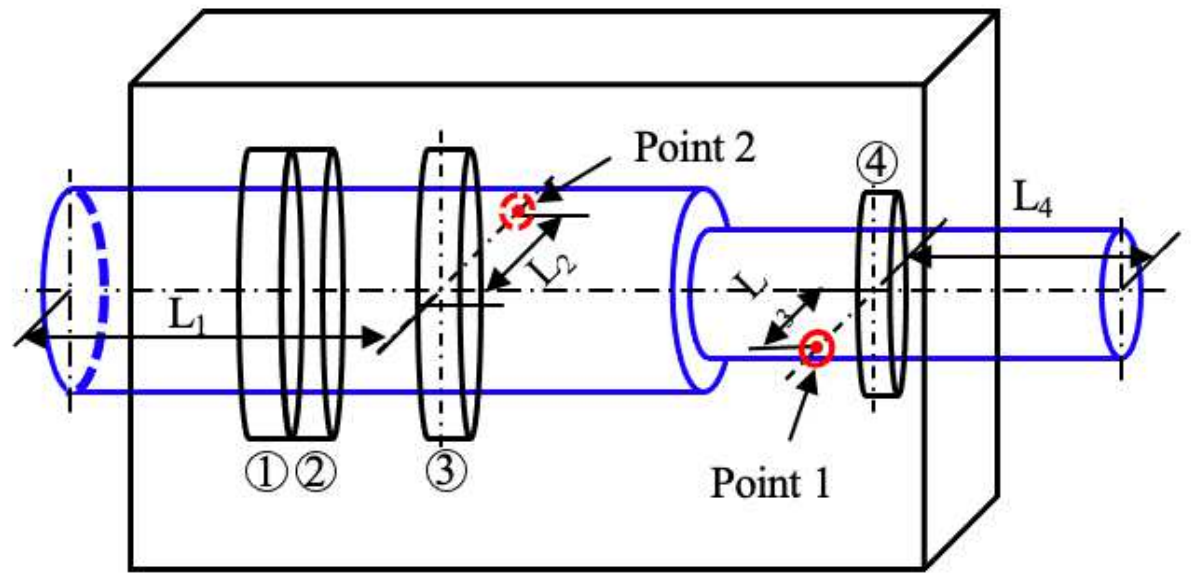

Figure 11

Temperature test rig and measuring positions
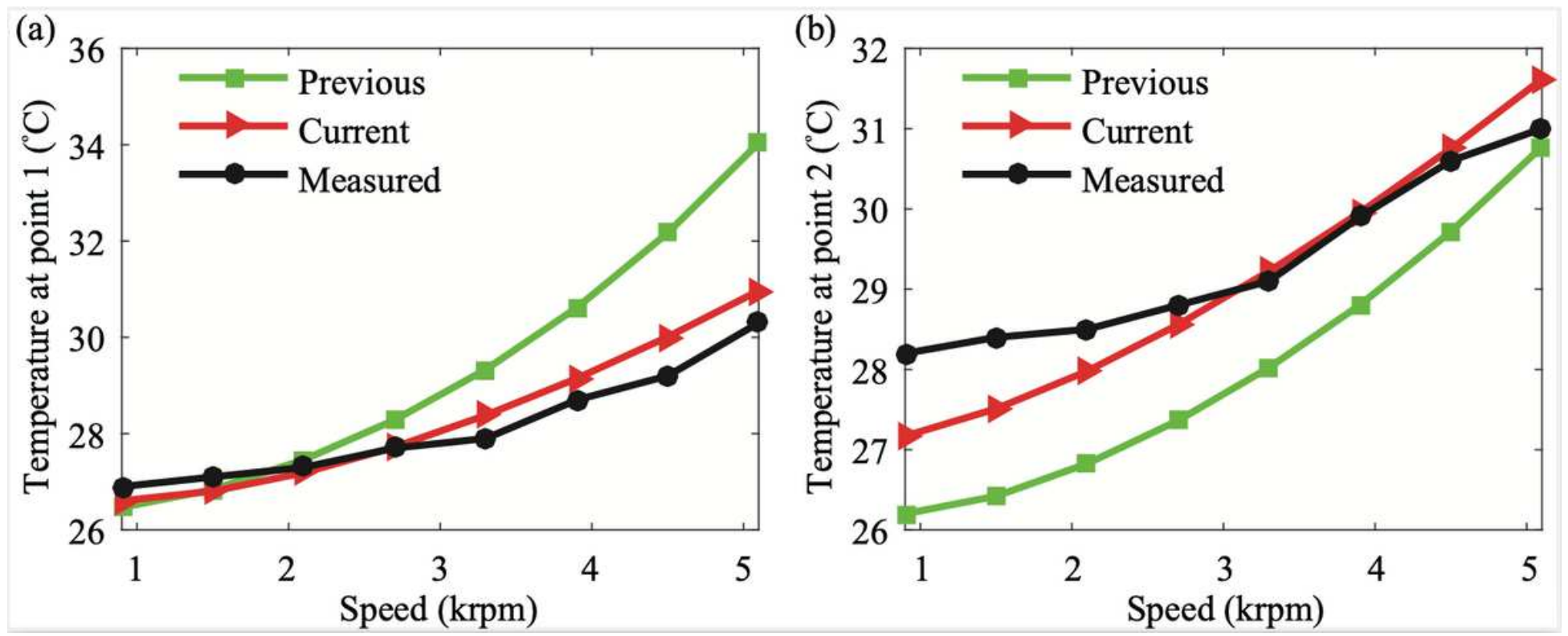

Figure 12 
The predicted and measured temperature at: (a) point 1 and (b) point 2
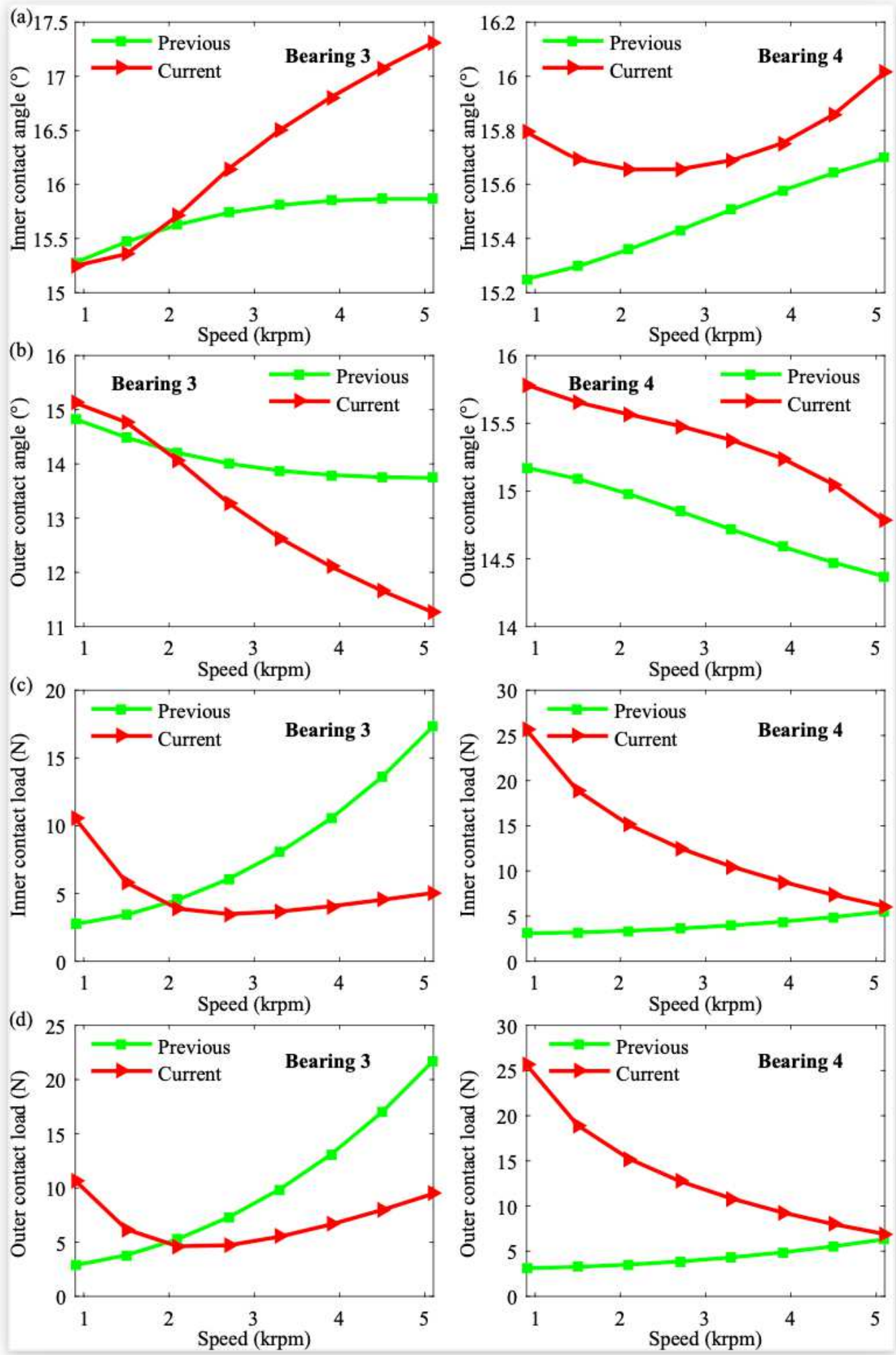

Figure 13

Contact angle and contact load of inner and outer rings 

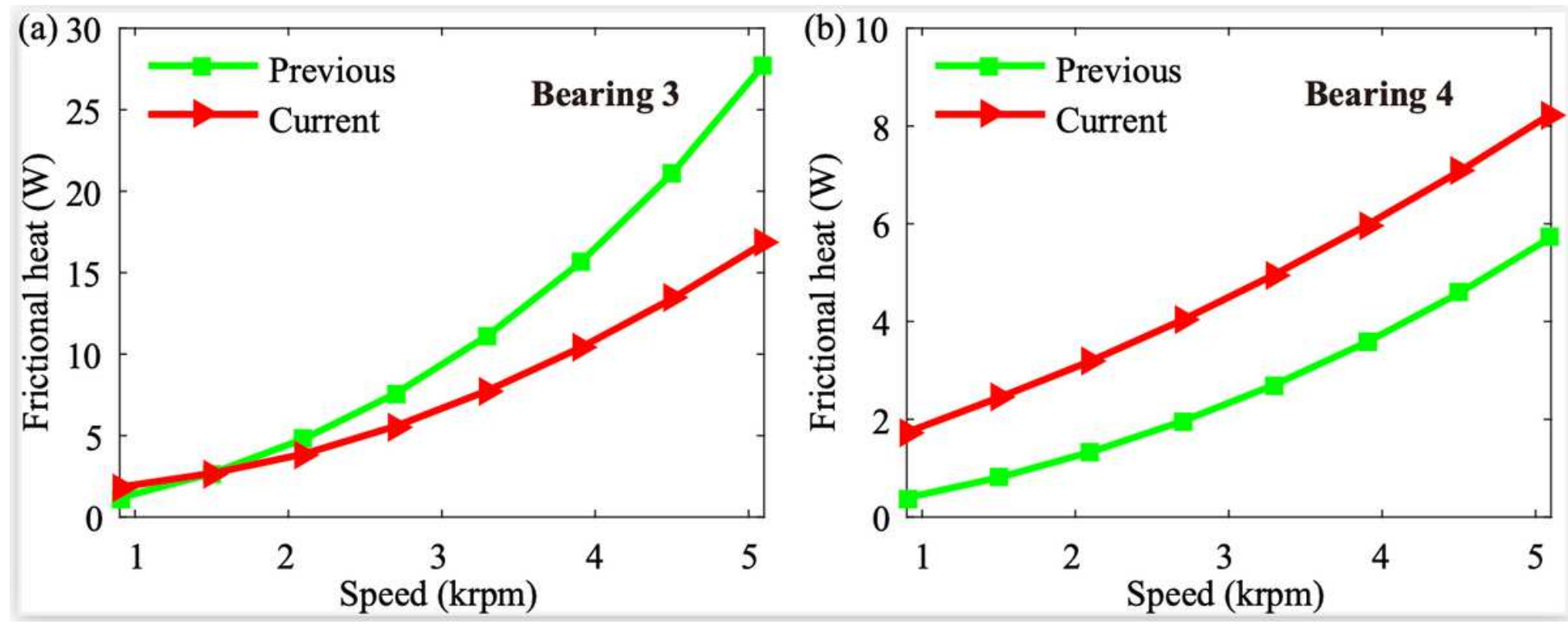

Figure 14

Frictional heat of bearings
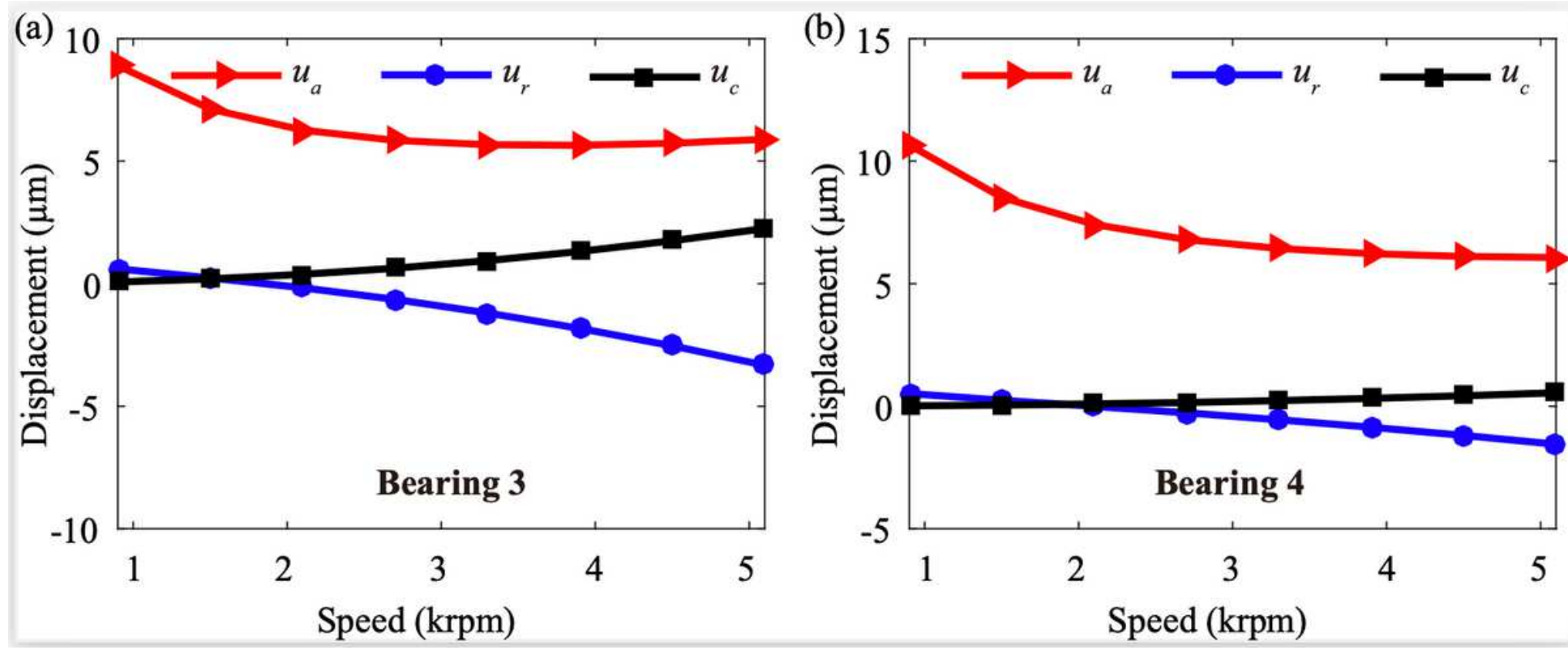

Figure 15

Thermal displacement and centrifugal displacement of bearings 3 and 4 